Effect of Tetrahydroquinoline Dyes Structure on the Performance of

\title{
Organic Dye Sensitized Solar Cells
}

Ruikui Chen, ${ }^{\dagger}$ Xichuan Yang, ${ }^{*}{ }^{\dagger}$ Haining Tian, ${ }^{\dagger}$ Xiuna Wang, ${ }^{\dagger}$ Anders Hagfeldt, ${ }^{\dagger}$ and Licheng $\operatorname{Sun}^{*}, \uparrow, \S$

${ }^{\dagger}$ State Key Laboratory of Fine Chemicals, DUT-KTH Joint Education and Research Center on Molecular Devices, Dalian University of Technology (DUT), Zhongshan Road 158-46, 116012 Dalian, P R China

${ }^{*}$ Center of Molecular Devices, Department of Chemistry, Physical Chemistry, Royal Institute of Technology, Teknikringen 30, 10044 Stockholm, Sweden

${ }^{\S}$ Center of Molecular Devices, Department of Chemistry, Organic Chemistry, Royal Institute of Technology (KTH), Teknikringen 30, 10044 Stockholm, Sweden

*Corresponding authors: Tel. +86-411-88993886, Fax. +86-411-83702185

Email: yangxc@dlut.edu.cn (Dr. X. Yang)

Tel. +46-8-7908127, Fax. +46-8-7912333

Email: lichengs@kth.se (Dr. L. Sun)

\section{Contents:}

(A) Synthesis and photophysical data for the intermediates and sensitizers:.........S2

(B) Photophysical properties of the tetrahydroquinoline sensitizers: .....................S20

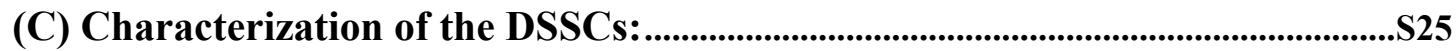

(D) Complete Reference 38 .....................................................................................................530 


\section{(A) Synthesis and photophysical data for the intermediates and sensitizers:}

General: ${ }^{1} \mathrm{H}$ NMR Spectra were recorded with a Varian INOVA 400NMR instrument. MS data were obtained with GCT CA156 (UK) high resolution mass spectrometer (HRMS) or HP1100 LC/MSD (USA) mass spectrometer. UV-vis spectra of the dyes in solutions were recorded in a quartz cell with $1 \mathrm{~cm}$ path length on a HP 8453 spectraphotometer. Melting-points were measured with a X-4 melting-point apparatus with microscope.

The several of $\pi$-conjugating spacers 5a-8a were prepared using known procedures. 5a and 6a were prepared by Suzuki coupling of 2-thienylboronic acid with corresponding 2-bromothiophene and 2,5-dibromothiophene, respectively. 7a was obtained in 3 steps from 3-bromothiophene according to the already reported procedures. $^{[1,2]} \mathbf{8 a}$ of E-configuration was prepared by McMurry coupling of 2-thiophenecarboxaldehyde. ${ }^{[3]}$

\section{2,2,4-Trimethyl-1,2-dihydro-quinoline(1a)}

To a solution of aniline (18.7 $\mathrm{g}, 0.2 \mathrm{~mol})$ and toluenesulfonic acid (1.9 $\mathrm{g})$ in cyclohexane $(20 \mathrm{~mL})$, acetone $(42 \mathrm{~mL}, 0.57 \mathrm{~mol})$ was added dropwise at $80 \sim 90{ }^{\circ} \mathrm{C}$ for $8 \sim 10 \mathrm{~h}$. The resulted water was removed by co-boiling with cyclohexane. Sodium carbonate $(0.55 \mathrm{~g})$ in water $(20 \mathrm{~mL})$ was poured into the reaction mixture after complete addition of acetone at $70{ }^{\circ} \mathrm{C}$. The reaction mixture was stirred overnight at r.t. The organic layer was washed with water and dried over magnesium sulfate. The unreacted acetone and solvent was romoved by rotary evaporation. The residue was distilled in vacuo to give $1 \mathrm{a}$ as colorless oil $\left(130{ }^{\circ} \mathrm{C} / 10 \mathrm{mmHg}, 17 \mathrm{~g}, 49 \%\right) .{ }^{1} \mathrm{H}$ NMR $\left(\mathrm{CDCl}_{3}, 400 \mathrm{MHz}, \mathrm{ppm}\right): \delta 1.27(\mathrm{~s}, 6 \mathrm{H}), 1.98(\mathrm{~d}, J=1.2 \mathrm{~Hz}, 3 \mathrm{H}), 3.66(\mathrm{~s}, 1 \mathrm{H}), 5.30(\mathrm{~d}$, $J=1.2 \mathrm{~Hz}, 1 \mathrm{H}), 6.43(\mathrm{~d}, J=7.8 \mathrm{~Hz}, 1 \mathrm{H}), 6.63\left(\mathrm{dd}, J_{1}=8.0 \mathrm{~Hz}, J_{2}=7.5 \mathrm{~Hz}, 1 \mathrm{H}\right)$, $6.97\left(\mathrm{dd}, J_{1}=J_{2}=7.6 \mathrm{~Hz}, 1 \mathrm{H}\right), 7.05(\mathrm{~d}, J=7.6 \mathrm{~Hz}, 1 \mathrm{H})$.

\section{2,2,4-Trimethyl-1,2,3,4-dihydro-quinoline (1b)}

To a solution of $1 \mathrm{a}(12.05 \mathrm{~g})$ in absolute ethanol was added a $50 \%(\mathrm{w} / \mathrm{w})$ slurry of Raney nickel in ethanol (1 g). The reaction was hydrogenated over $\mathrm{H}_{2}$ gas (1 MPa) at $130{ }^{\circ} \mathrm{C}$. The resulting solution was filtered carefully over celite and washed with 
ethanol. The solvent was removed in vacuo and the residue (12 g, 99\%) was used for the next reaction without more purification. ${ }^{1} \mathrm{H} \mathrm{NMR}\left(\mathrm{CDCl}_{3}, 400 \mathrm{MHz}, \mathrm{ppm}\right): \delta 1.15$ (s, 3H), $1.21(\mathrm{~s}, 3 \mathrm{H}), 1.32(\mathrm{~d}, J=6.7 \mathrm{~Hz}, 3 \mathrm{H}), 1.43\left(\mathrm{dd}, J_{1}=12.5 \mathrm{~Hz}, J_{2}=12.6 \mathrm{~Hz}\right.$, $1 \mathrm{H}), 1.71\left(\mathrm{dd}, J_{1}=5.6 \mathrm{~Hz}, J_{2}=12.9 \mathrm{~Hz}, 1 \mathrm{H}\right), 2.87-2.93(\mathrm{~m}, 1 \mathrm{H}), 3.53(\mathrm{~s}, 1 \mathrm{H}), 6.41(\mathrm{~d}$, $J=7.9 \mathrm{~Hz}, 1 \mathrm{H}), 6.64\left(\mathrm{dd}, J_{1}=7.5 \mathrm{~Hz}, J_{2}=7.4 \mathrm{~Hz}, 1 \mathrm{H}\right), 6.95\left(\mathrm{dd}, J_{1}=7.8 \mathrm{~Hz}, J_{2}=\right.$ $7.4 \mathrm{~Hz}, 1 \mathrm{H}), 7.14(\mathrm{~d}, J=7.6 \mathrm{~Hz}, 1 \mathrm{H})$.

\section{1,2,2,4-Tetramethyl-1,2,3,4-dihydro-quinoline (1c)}

$1 \mathbf{b}(1.75 \mathrm{~g}, 10 \mathrm{mmol})$ and $\left(\mathrm{CH}_{3}\right)_{2} \mathrm{SO}_{4}(0.76 \mathrm{~g}, 6 \mathrm{mmol})$ were dissolved in benzene. The mixture was refluxed for $2 \mathrm{~h} .10 \% \mathrm{NH}_{4} \mathrm{OH}(10 \mathrm{~mL})$ was added at $70{ }^{\circ} \mathrm{C}$ and stirred for $1 \mathrm{~h}$. The organic layer was dried over magnesium sulfate. The solvent was removed by rotary evaporation and the residue was purified by chromatography ( silica gel, dichloromethane: hexane $=1: 1)$ to provide $1.5 \mathrm{~g}(79 \%)$ of the title product as colorless oil. ${ }^{1} \mathrm{H} \mathrm{NMR}\left(\mathrm{CDCl}_{3}, 400 \mathrm{MHz}, \mathrm{ppm}\right): \delta 1.18(\mathrm{~s}, 3 \mathrm{H}), 1.25(\mathrm{~s}, 3 \mathrm{H}), 1.32(\mathrm{~d}$, $J=6.7 \mathrm{~Hz}, 3 \mathrm{H}), 1.51\left(\mathrm{dd}, J_{1}=12.7 \mathrm{~Hz}, J_{2}=12.8 \mathrm{~Hz}, 1 \mathrm{H}\right), 1.75\left(\mathrm{dd}, J_{1}=4.5 \mathrm{~Hz}, J_{2}=\right.$ $12.9 \mathrm{~Hz}, 1 \mathrm{H}), 2.78(\mathrm{~s}, 3 \mathrm{H}), 2.80-2.86(\mathrm{~m}, 1 \mathrm{H}), 6.56(\mathrm{~d}, J=8.1 \mathrm{~Hz}, 1 \mathrm{H}), 6.65\left(\mathrm{dd}, J_{l}=\right.$ $\left.7.4 \mathrm{~Hz}, J_{2}=8.2 \mathrm{~Hz}, 1 \mathrm{H}\right), 7.06-7.12(\mathrm{~m}, 2 \mathrm{H})$.

6-Formyl-1,2,2,4-tetramethyl-1,2,3,4-dihydro-quinoline (1d)

$\mathrm{POCl}_{3}(0.49 \mathrm{~mL}, 5.4 \mathrm{mmol})$ was added dropwise with stirring to a solution of $\mathbf{1 c}$ $(1.008 \mathrm{~g}, 5.3 \mathrm{mmol})$ in fresh distilled DMF $(1.3 \mathrm{~mL}, 16.7 \mathrm{mmol})$ at $15 \sim 20{ }^{\circ} \mathrm{C}$ under $\mathrm{N}_{2}$ protect. Then the mixture was held at $55^{\circ} \mathrm{C}$ for $6 \mathrm{~h}$ and then cooled, ice $(100 \mathrm{~g})$ was added, and then $5 \mathrm{~N} \mathrm{NaOH}$ to $\mathrm{pH}$ 6. The precipitate was filtered, dried under reduced pressure to give $\mathbf{1 d}$ as green solid $(631 \mathrm{mg}, 55 \%) .{ }^{1} \mathrm{H} \mathrm{NMR}\left(\mathrm{CDCl}_{3}, 400 \mathrm{MHz}\right.$, ppm): $\delta 1.28(\mathrm{~s}, 3 \mathrm{H}), 1.34(\mathrm{~s}, 3 \mathrm{H}), 1.40(\mathrm{~d}, J=6.6 \mathrm{~Hz}, 3 \mathrm{H}), 1.54\left(\mathrm{dd}, J_{l}=13.0 \mathrm{~Hz}, J_{2}\right.$ $=13.1 \mathrm{~Hz}, 1 \mathrm{H}), 1.83\left(\mathrm{dd}, J_{1}=4.5 \mathrm{~Hz}, J_{2}=13.1 \mathrm{~Hz}, 1 \mathrm{H}\right), 2.92(\mathrm{~s}, 3 \mathrm{H}), 2.82-2.90(\mathrm{~m}$, $1 \mathrm{H}), 6.59(\mathrm{~d}, J=8.5 \mathrm{~Hz}, 1 \mathrm{H}), 7.59$ (d, $J=8.6 \mathrm{~Hz}, 1 \mathrm{H}), 7.64(\mathrm{~s}, 1 \mathrm{H}), 9.71$ (s, 1H).

6-Bromo-1,2,2,4-tetramethyl-1,2,3,4-dihydro-quinoline(1e)

1c $(2.54 \mathrm{~g}, 13.4 \mathrm{mmol})$ and NBS $(2.63 \mathrm{~g}, 14.7 \mathrm{mmol})$ were dissolved in $20 \mathrm{~mL} \mathrm{CCl}_{4}$. This solution was stirred at r. t. for $2 \mathrm{~h}$. The solvent was removed by rotary evaporation and the residue was purified by chromatography (silica gel, dichloromethane: hexane =1:2). 1e (3.36g, 93\%) was obtained as colorless solid. Mp. 
37-46 ${ }^{\circ} \mathrm{C} .{ }^{1} \mathrm{H}$ NMR $\left(\mathrm{CDCl}_{3}, 400 \mathrm{MHz}, \mathrm{ppm}\right): \delta 1.17$ (s, 3H), 1.25 (s, 3H), 1.30 (d, $J=$ $6.7 \mathrm{~Hz}, 3 \mathrm{H}), 1.47\left(\mathrm{dd}, J_{1}=J_{2}=12.8 \mathrm{~Hz}, 1 \mathrm{H}\right), 1.75\left(\mathrm{dd}, J_{l}=4.5 \mathrm{~Hz}, J_{2}=13.0 \mathrm{~Hz}, 1 \mathrm{H}\right)$, $2.75(\mathrm{~s}, 3 \mathrm{H}), 2.78-2.80(\mathrm{~m}, 1 \mathrm{H}), 6.41(\mathrm{~d}, J=8.6 \mathrm{~Hz}, 1 \mathrm{H}), 7.15\left(\mathrm{dd}, J_{l}=2.3 \mathrm{~Hz}, J_{2}=\right.$ $8.7 \mathrm{~Hz}, 1 \mathrm{H}), 7.17(\mathrm{~s}, 1 \mathrm{H})$.

\section{1,2,2,4-Tetramethyl-1,2,3,4-dihydro-quinoline-6-boronic acid (1f)}

To a THF $(20 \mathrm{~mL})$ solution of 1 e $(540 \mathrm{mg}, 2 \mathrm{mmol})$ was added a hexane solution of $n$-BuLi $(2.57 \mathrm{M}, 0.85 \mathrm{~mL}, 2.3 \mathrm{mmol})$ at $-78{ }^{\circ} \mathrm{C}$. After being stirred for $1 \mathrm{~h}$ at this temperature, $\mathrm{B}(\mathrm{OBu})_{3}(1 \mathrm{~mL}, 3.7 \mathrm{mmol})$ was added dropwise to this solution. After the mixture was stirred for another $1 \mathrm{~h}$ at $-78{ }^{\circ} \mathrm{C}$, it was warmed to ambient temperature and stirred overnight. The solution was partitioned between saturated aqueous $\mathrm{NH}_{4} \mathrm{Cl}$ $(50 \mathrm{~mL})$ and ethyl acetate $(50 \mathrm{~mL})$. The aqueous layer was extracted further with dichloromethane $(3 \times 20 \mathrm{~mL})$ and the combined organic layers were dried over $\mathrm{MgSO}_{4}$. The product was purified by flash chromatography (silica gel, $\mathrm{CH}_{2} \mathrm{Cl}_{2}: \mathrm{MeOH}=40: 1$ ) to provide the boronic acid as white solid (230mg, 50\%). ${ }^{1} \mathrm{H} \mathrm{NMR}\left(\mathrm{CDCl}_{3}, 400 \mathrm{MHz}\right.$, ppm): $\delta 1.25$ (s, 3H), $1.33(\mathrm{~s}, 3 \mathrm{H}), 1.46(\mathrm{~d}, J=6.6 \mathrm{~Hz}, 3 \mathrm{H}), 1.59\left(\mathrm{dd}, J_{l}=12.5 \mathrm{~Hz}, J_{2}\right.$ $=13.0 \mathrm{~Hz}, 1 \mathrm{H}), 1.82\left(\mathrm{dd}, J_{l}=4.3 \mathrm{~Hz}, J_{2}=12.9 \mathrm{~Hz}, 1 \mathrm{H}\right), 2.88-2.90(\mathrm{~m}, 4 \mathrm{H}), 6.66(\mathrm{~d}, J$ $=7.8 \mathrm{~Hz}, 3 \mathrm{H}), 7.96-7.80(\mathrm{~m}, 2 \mathrm{H})$.

(1,2,2,4-Tetramethyl-1,2,3,4-tetrahydro-quinolin-6-yl)-methanol (1g) and (1,2,2,4-Tetramethyl-1,2,3,4-tetrahydro-quinolin-6-yl-methyl)triphenylphosphonium bromide (1h)

To a solution of aldehyde 1d $(555 \mathrm{mg}, 2.56 \mathrm{mmol})$ in absolute EtOH $(10 \mathrm{~mL})$, an excess of $\mathrm{NaBH}_{4}$ was added portionwise until consumption of the starting material as followed by TLC $\left(\mathrm{CH}_{2} \mathrm{Cl}_{2}\right)$. After dilution with $\mathrm{Et}_{2} \mathrm{O}$, the mixture was hydrolyzed using $2 \mathrm{~mL} \mathrm{H}_{2} \mathrm{O}$. The organic phase was dried over $\mathrm{Na}_{2} \mathrm{SO}_{4}$ and evaporated in vacuo to give the corresponding alcohol $\mathbf{1 g}$ which was directively dissolved in $\mathrm{CHCl}_{3}(20 \mathrm{~mL})$ in the presence of $\mathrm{HPPh}_{3} \mathrm{Br}(0.99 \mathrm{~g}, 2.86 \mathrm{mmol})$ (prepared from a solution of $\mathrm{PPh}_{3}$ and gaseous $\mathrm{HBr}) .{ }^{[4]}$ The reaction mixture was refluxed for $3 \mathrm{~h}$, and the solvent was evaporated after cooling. The residue was purified by chromatography (silica gel, $\left.\mathrm{CH}_{2} \mathrm{Cl}_{2}: \mathrm{MeOH}=20: 1\right)$ and further triturated in the minimum of $\mathrm{Et}_{2} \mathrm{O}$ leading to the precipitate of a white solid which was filtered to give a white powder $\mathbf{1 h}$ (510mg, 
37\%). Mp. $190-191{ }^{\circ} \mathrm{C} .{ }^{1} \mathrm{H}$ NMR $\left(\mathrm{CDCl}_{3}, 400 \mathrm{MHz}, \mathrm{ppm}\right): \delta 0.90$ (d, $\left.J=6.6 \mathrm{~Hz}, 3 \mathrm{H}\right)$, $1.12(\mathrm{~s}, 3 \mathrm{H}), 1.25(\mathrm{~s}, 3 \mathrm{H}), 1.37\left(\mathrm{dd}, J_{1}=13.0 \mathrm{~Hz}, J_{2}=13.1 \mathrm{~Hz}, 1 \mathrm{H}\right), 1.69\left(\mathrm{dd}, J_{1}=4.5\right.$ $\left.\mathrm{Hz}, J_{2}=12.8 \mathrm{~Hz}, 1 \mathrm{H}\right), 2.61-2.64(\mathrm{~m}, 1 \mathrm{H}), 2.72(\mathrm{~s}, 3 \mathrm{H}), 4.97\left(\mathrm{dd}, J_{1}=13.4 \mathrm{~Hz}, J_{2}=\right.$ $14.5 \mathrm{~Hz}, 1 \mathrm{H}), 5.09$ (dd, $\left.J_{1}=13.5 \mathrm{~Hz}, J_{2}=14.4 \mathrm{~Hz}, 1 \mathrm{H}\right), 6.34(\mathrm{~d}, J=8.1 \mathrm{~Hz}, 1 \mathrm{H}), 6.62$ (s, 1H), $6.78(\mathrm{~d}, J=8.1 \mathrm{~Hz}, 1 \mathrm{H}), 7.64-7.79(\mathrm{~m}, 15 \mathrm{H})$. MS: API-ES positive: [quinoline- $\left.\mathrm{PPh}_{3}{ }^{+}\right](\mathrm{m} / \mathrm{z}=464.5)$; API-ES negative: $\left[\mathrm{Br}^{-}\right](\mathrm{m} / z=79$ and 81$)$.

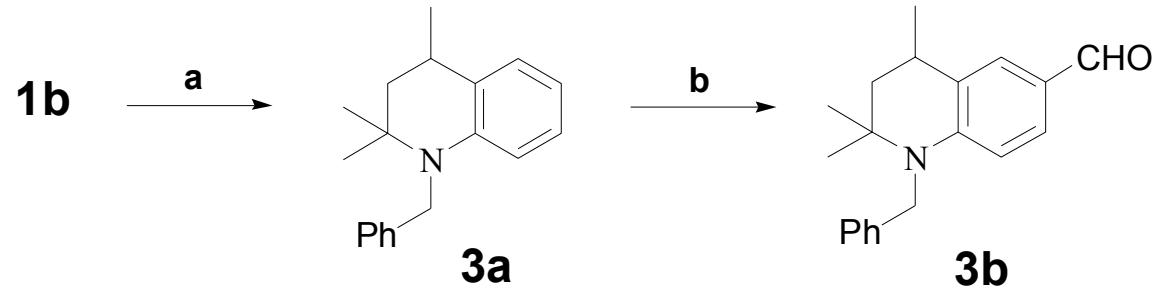

Scheme S1 Synthesis route of 3d. a) i. $n$-BuLi, THF, $-15{ }^{\circ} \mathrm{C}$; ii. benzyl bromide, $4 \mathrm{~h}, 86 \%$; b) $\mathrm{POCl}_{3} / \mathrm{DMF}, \mathrm{CH}_{2} \mathrm{ClCH}_{2} \mathrm{Cl}$, reflux, 4h, 94\%.

\section{1-Benzyl-2,2,4-trimethyl-1,2,3,4-tetrahydro-quinoline (3a)}

To a solution of $\mathbf{1 b}(1.796 \mathrm{~g}, 10.3 \mathrm{mmole})$ in absolute THF $(40 \mathrm{~mL})$ was added a solution of $n$-BuLi in hexane $(2.57 \mathrm{M}, 4.4 \mathrm{~mL}, 11.3 \mathrm{mmole})$ at $-15^{\circ} \mathrm{C}$. After stirring for $30 \mathrm{~min}$ at this temperature, benzyl bormide $(1.35 \mathrm{~mL}, 11.3 \mathrm{mmol})$ was added dropwise to this solution. The reaction mixture was stirred for another $4 \mathrm{~h}$ and the solvent was removed by rotary evaporator. After purification by chromatography (silica gel, $\mathrm{CH}_{2} \mathrm{Cl}_{2}$ :hexane=1:5), the product was obtained as colorless oil $(2.343 \mathrm{~g}, 86 \%) .{ }^{1} \mathrm{H}$ NMR ( $\left.\mathrm{CDCl}_{3}, 400 \mathrm{MHz}, \mathrm{ppm}\right): \delta 1.25$ (s, 3H), 1.27 (s, 3H), $1.38(\mathrm{~d}, J=6.6 \mathrm{~Hz}, 3 \mathrm{H})$, $1.76\left(\mathrm{dd}, J_{1}=12.6 \mathrm{~Hz}, J_{2}=12.8 \mathrm{~Hz}, 1 \mathrm{H}\right), 1.83\left(\mathrm{dd}, J_{1}=5.2 \mathrm{~Hz}, J_{2}=13.0 \mathrm{~Hz}, 1 \mathrm{H}\right)$, 3.03-3.09 (m, 1H), $4.24(\mathrm{~d}, J=17.9 \mathrm{~Hz}, 1 \mathrm{H}), 4.70$ (d, $J=17.8 \mathrm{~Hz}, 1 \mathrm{H}), 6.30(\mathrm{~d}, J=$ $8.3 \mathrm{~Hz}, 1 \mathrm{H}), 6.62\left(\mathrm{dd}, J_{1}=7.3 \mathrm{~Hz}, J_{2}=7.4 \mathrm{~Hz}, 1 \mathrm{H}\right), 6.91\left(\mathrm{dd}, J_{1}=7.5 \mathrm{~Hz}, J_{2}=8.0 \mathrm{~Hz}\right.$, $1 \mathrm{H}), 7.17-7.22(\mathrm{~m}, 2 \mathrm{H}), 7.29-7.30(\mathrm{~m}, 4 \mathrm{H})$. HRMS-EI $(\mathrm{m} / \mathrm{z})$ : $[\mathrm{M}]^{+}$calcd for $\mathrm{C}_{19} \mathrm{H}_{23} \mathrm{~N}$, 265.1830; found, 265.1826.

1-Benzyl-6-formyl-2,2,4-trimethyl-1,2,3,4-tetrahydro-quinoline (3b)

To a solution of compound 3a $(927 \mathrm{mg}, 3.5 \mathrm{mmol})$ and anhydrous DMF $(0.4 \mathrm{~mL}$, $5.2 \mathrm{mmol})$ in anhydrous 1,2-dichloroethane $(10 \mathrm{~mL})$ was added $\mathrm{POCl}_{3}(0.5 \mathrm{~mL}$, $5.3 \mathrm{mmol}$ ), and the mixture was refluxed for $4 \mathrm{~h}$. After cooling, the mixture was 
poured into ice water $(50 \mathrm{~mL})$ and stirred for $2 \mathrm{~h}$. After neutralization using aqueous solution of sodium hydroxy $(1 \mathrm{M})$, the aquesous phase was extracted with $\mathrm{CH}_{2} \mathrm{Cl}_{2}$ $(3 \times 30 \mathrm{~mL})$. The organic fraction were gathered, dried over $\mathrm{MgSO}_{4}$, and evaporated in vacuo. After chromatography on silica gel $\left(\mathrm{CH}_{2} \mathrm{Cl}_{2}\right)$, green solid 3b (964 mg, 94 \%) were obtained. Mp. $86-87^{\circ} \mathrm{C} .{ }^{1} \mathrm{H}$ NMR $\left(\mathrm{CDCl}_{3}, 400 \mathrm{MHz}, \mathrm{ppm}\right): \delta 1.31$ (s, 3H), 1.32 $(\mathrm{s}, 3 \mathrm{H}), 1.44(\mathrm{~d}, J=6.2 \mathrm{~Hz}, 3 \mathrm{H}), 1.76\left(\mathrm{dd}, J_{1}=12.9 \mathrm{~Hz}, J_{2}=13.0 \mathrm{~Hz}, 1 \mathrm{H}\right), 1.92(\mathrm{dd}$, $\left.J_{l}=4.1 \mathrm{~Hz}, J_{2}=13.0 \mathrm{~Hz}, 1 \mathrm{H}\right), 4.43(\mathrm{~d}, J=17.9 \mathrm{~Hz}, 1 \mathrm{H}), 4.77(\mathrm{~d}, J=17.9 \mathrm{~Hz}, 1 \mathrm{H})$, $6.35(\mathrm{~d}, J=8.4 \mathrm{~Hz}, 1 \mathrm{H}), 7.21-7.25(\mathrm{~m}, 3 \mathrm{H}), 7.31(\mathrm{~d}, J=6.9 \mathrm{~Hz}, 2 \mathrm{H}), 7.40(\mathrm{~d}, J=8.2$ $\mathrm{Hz}, 1 \mathrm{H}), 7.71(\mathrm{~s}, 1 \mathrm{H}), 9.67(\mathrm{~s}, 1 \mathrm{H})$. HRMS-EI $(\mathrm{m} / \mathrm{z}):[\mathrm{M}]^{+}$calcd for $\mathrm{C}_{20} \mathrm{H}_{23} \mathrm{NO}$, 293.1780; found, 293.1782.

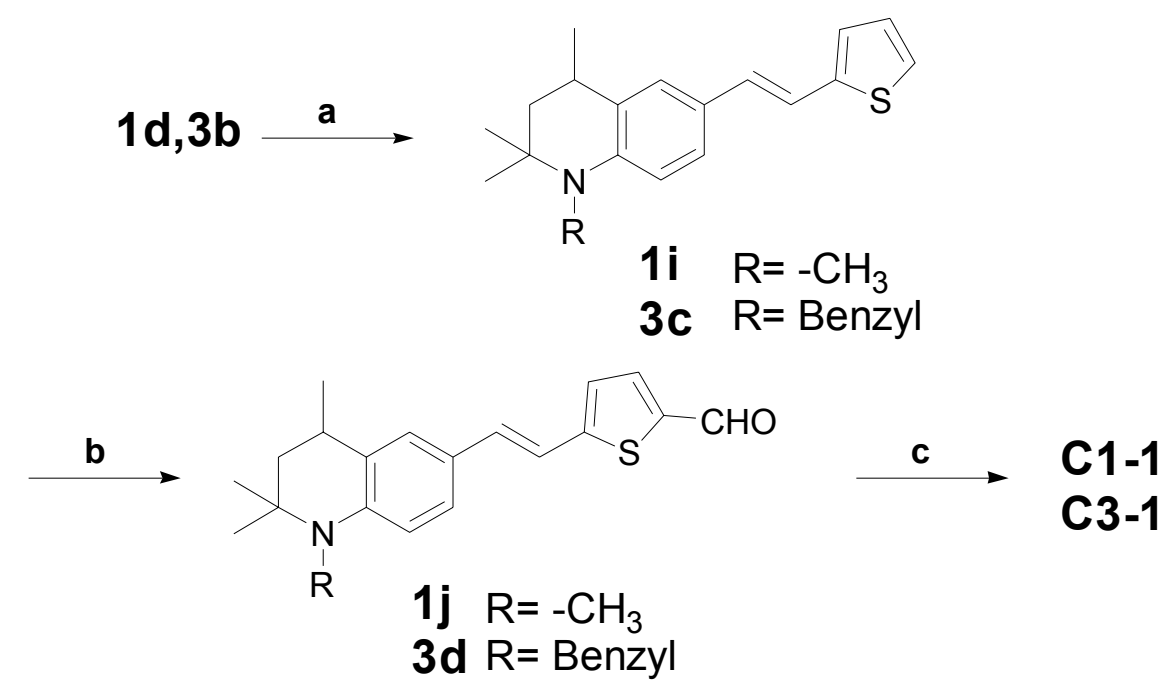

Scheme S2 Synthesis Route of C1-1 and C3-1 dyes. a) diethyl 2-thienylmethylphosphonate, $t$-BuOK, THF, $0{ }^{\circ} \mathrm{C}, 2 \mathrm{~h}$; b) i. $n$-BuLi, THF, $-15{ }^{\circ} \mathrm{C}, 20 \mathrm{~min}$; ii. DMF, $-15{ }^{\circ} \mathrm{C}, 2 \mathrm{~h}$; iii. water; c) cyano-acetic acid, $\mathrm{CH}_{3} \mathrm{CN}$, piperidine, reflux for $2 \mathrm{~h}$.

\section{1,2,2,4-Tetramethyl-6-(2-thiphen-2-yl-vinyl)-1,2,3,4-tetrahydro-quinoline (1i)}

To a solution of $t$-BuOK (353 mg, $3.15 \mathrm{mmol})$ in $10 \mathrm{~mL}$ fresh distilled THF, diethyl 2-thienylmethylphosphonate $(725 \mathrm{mg}, 3.1 \mathrm{mmol})$ was added dropwise at $0{ }^{\circ} \mathrm{C}$ under protection of $\mathrm{N}_{2}$. The mixture was vigorously stirred in an ice bath. Then $\mathbf{1 d}(545 \mathrm{mg}$, $2.5 \mathrm{mmol}$ ) in $10 \mathrm{~mL}$ anhydrated THF was added dropwise in about $30 \mathrm{~min}$ and stirring was continued for $2 \mathrm{~h}$. After removing the solvent, the residue was loaded on column chromatography (silica gel, $\mathrm{CH}_{2} \mathrm{Cl}_{2}$ as eluent). The desired product was obtained as a 
yellow solid (706 mg, 95 \%). Mp. 87-89 ${ }^{\circ} \mathrm{C} .{ }^{1} \mathrm{H}$ NMR (Acetone-d 6 , 400MHz, ppm): $\delta$ $1.21(\mathrm{~s}, 3 \mathrm{H}), 1.30(\mathrm{~s}, 3 \mathrm{H}), 1.36(\mathrm{~d}, J=6.7 \mathrm{~Hz}, 3 \mathrm{H}), 1.46\left(\mathrm{dd}, J_{1}=12.7 \mathrm{~Hz}, J_{2}=13.0\right.$ $\mathrm{Hz}, 1 \mathrm{H}), 1.85\left(\mathrm{dd}, J_{1}=4.5 \mathrm{~Hz}, J_{2}=12.9 \mathrm{~Hz}, 1 \mathrm{H}\right), 2.84(\mathrm{~s}, 3 \mathrm{H}), 2.80-2.87(\mathrm{~m}, 1 \mathrm{H})$, $6.56(\mathrm{~d}, J=8.5 \mathrm{~Hz}, 1 \mathrm{H}), 6.88(\mathrm{~d}, J=16.0 \mathrm{~Hz}, 1 \mathrm{H}), 6.99\left(\mathrm{dd}, J_{1}=3.5 \mathrm{~Hz}, J_{2}=5.1 \mathrm{~Hz}\right.$, 1H), 7.04 (d, $J=3.5 \mathrm{~Hz}, 1 \mathrm{H}), 7.15$ (d, $J=16.1 \mathrm{~Hz}, 1 \mathrm{H}), 7.23-7.24$ (m, 2H), 7.31 (s, 1H). HRMS-EI (m/z): [M] calcd for $\mathrm{C}_{19} \mathrm{H}_{23} \mathrm{NS}, 297.1551$; found, 297.1546.

5-[2-(1,2,2,4-Tetramethyl-1,2,3,4-tetrahydro-quinolin-6-yl)-vinyl]-thiophene-2-carb aldehyde (1j)

A solution of $n$-BuLi in hexane $(2.57 \mathrm{M}, 0.43 \mathrm{~mL}, 1.1 \mathrm{mmol})$ was added dropwise to a solution of $1 \mathbf{i}(297 \mathrm{mg}, 1 \mathrm{mmol})$ in anhydrated THF $(20 \mathrm{~mL})$ at $-15{ }^{\circ} \mathrm{C}$ under $\mathrm{N}_{2}$ protection. Then the mixture was stirred for another $20 \mathrm{~min}$ at $-15^{\circ} \mathrm{C}$. Anhydrous DMF $(0.09 \mathrm{~mL}, 1.2 \mathrm{mmol})$ was added to the above reaction mixture. After stirring at $-15^{\circ} \mathrm{C}$ for $2 \mathrm{~h}$, the reaction mixture was poured into $50 \mathrm{~mL}$ of ice water. After extracted three times by $\mathrm{CH}_{2} \mathrm{Cl}_{2}$, the extraction was dried on magnesium sulfate and removed of solvent. The residue was subjected to column chromatography (silica gel, $\mathrm{CH}_{2} \mathrm{Cl}_{2}$ as the eluent), $182 \mathrm{mg}(56 \%)$ of red-brown solid was obtained. Mp. 138-139 ${ }^{\circ} \mathrm{C} .{ }^{1} \mathrm{H}$ NMR (Acetone-d $\left.6,400 \mathrm{MHz}, \mathrm{ppm}\right): \delta 1.24$ (s, 3H), 1.32 (s, 3H), 1.37 (d, $J=$ $6.6 \mathrm{~Hz}, 3 \mathrm{H}), 1.47\left(\mathrm{dd}, J_{1}=12.7 \mathrm{~Hz}, J_{2}=12.9 \mathrm{~Hz}, 1 \mathrm{H}\right), 1.87\left(\mathrm{dd}, J_{1}=4.4 \mathrm{~Hz}, J_{2}=\right.$ 13.0 Hz, 1H), 2.88 (s, 3H), 2.79-2.85 (m, 1H), 6.59 (d, $J=8.6 \mathrm{~Hz}, 1 \mathrm{H}), 7.20-7.22$ (m, $3 \mathrm{H}), 7.33\left(\mathrm{dd}, J_{1}=1.7 \mathrm{~Hz}, J_{2}=8.5 \mathrm{~Hz}, 1 \mathrm{H}\right), 7.39(\mathrm{~s}, 1 \mathrm{H}), 7.82(\mathrm{~d}, J=3.9 \mathrm{~Hz}, 1 \mathrm{H})$, 9.85 (s, 1H); HRMS-EI (m/z): [M] $]^{+}$calcd for $\mathrm{C}_{20} \mathrm{H}_{23} \mathrm{NOS}$, 325.1500; found, 325.1500 . 1-Benzyl-2,2,4-trimethyl-6-(2-thiophen-2-yl-vinyl)-1,2,3,4-tetrahydro-quinoline (3c)

3c was prepared by the similar procedure of synthesizing 1i. Yellow solid (yield 70 \%). Mp. 119-120 ${ }^{\circ} \mathrm{C} .{ }^{1} \mathrm{H}$ NMR (Acetone-d 6 , 400MHz, ppm): $\delta 1.31$ (s, 3H), 1.32 (s, $3 \mathrm{H}), 1.42(\mathrm{~d}, J=6.6 \mathrm{~Hz}, 3 \mathrm{H}), 1.71\left(\mathrm{dd}, J_{l}=12.7 \mathrm{~Hz}, J_{2}=13.0 \mathrm{~Hz}, 1 \mathrm{H}\right), 1.95\left(\mathrm{dd}, J_{l}=\right.$ $\left.4.7 \mathrm{~Hz}, J_{2}=12.9 \mathrm{~Hz}, 1 \mathrm{H}\right), 3.04-3.06(\mathrm{~m}, 4 \mathrm{H}), 4.32(\mathrm{~d}, J=18.2 \mathrm{~Hz}, 1 \mathrm{H}), 4.82(\mathrm{~d}, J=$ $18.3 \mathrm{~Hz}, 1 \mathrm{H}), 6.27(\mathrm{~d}, J=8.7 \mathrm{~Hz}, 1 \mathrm{H}), 6.84(\mathrm{~d}, J=16.1 \mathrm{~Hz}, 1 \mathrm{H}), 6.99\left(\mathrm{dd}, J_{1}=3.5\right.$ $\left.\mathrm{Hz}, J_{2}=5.1 \mathrm{~Hz}, 1 \mathrm{H}\right), 7.02(\mathrm{~d}, J=2.7 \mathrm{~Hz}, 1 \mathrm{H}), 7.07\left(\mathrm{dd}, J_{1}=1.7 \mathrm{~Hz}, J_{2}=9.0 \mathrm{~Hz}, 1 \mathrm{H}\right)$, $7.13(\mathrm{~d}, J=16.3 \mathrm{~Hz}, 1 \mathrm{H}), 7.20-7.25(\mathrm{~m}, 2 \mathrm{H}), 7.31-7.32(\mathrm{~m}, 4 \mathrm{H}), 7.37(\mathrm{~s}, 1 \mathrm{H})$. HRMS-EI $(m / z):[M]^{+}$calcd for $\mathrm{C}_{25} \mathrm{H}_{27} \mathrm{NS}, 373.1864$; found, 373.1854 . 
5-[2-(1-Benzyl-2,2,4-trimethyl-1,2,3,4-tetrahydro-quinolin-6-yl)-vinyl]-thiophene-2-c arbaldehyde (3d)

3d was prepared by the similar procedure of synthesizing 1j. Orange solid (yield 65 \%). Mp. 121-122 ${ }^{\circ} \mathrm{C} .{ }^{1} \mathrm{H}$ NMR (Acetone- $\left.\mathrm{d}_{6}, 400 \mathrm{MHz}, \mathrm{ppm}\right): \delta 1.32$ (s, 3H), 1.33 (s, $3 \mathrm{H}), 1.43(\mathrm{~d}, J=6.5 \mathrm{~Hz}, 3 \mathrm{H}), 1.72\left(\mathrm{dd}, J_{1}=12.8 \mathrm{~Hz}, J_{2}=13.0 \mathrm{~Hz}, 1 \mathrm{H}\right), 1.96\left(\mathrm{dd}, J_{1}=\right.$ $\left.4.7 \mathrm{~Hz}, J_{2}=13.3 \mathrm{~Hz}, 1 \mathrm{H}\right), 3.04-3.07(\mathrm{~m}, 4 \mathrm{H}), 4.37(\mathrm{~d}, J=18.4 \mathrm{~Hz}, 1 \mathrm{H}), 4.85(\mathrm{~d}, J=$ $18.4 \mathrm{~Hz}, 1 \mathrm{H}), 6.31(\mathrm{~d}, J=8.9 \mathrm{~Hz}, 1 \mathrm{H}), 7.16-7.23(\mathrm{~m}, 5 \mathrm{H}), 7.30-7.32(\mathrm{~m}, 4 \mathrm{H}), 7.46$ (s, 1H), $7.83(\mathrm{~d}, J=4.0 \mathrm{~Hz}, 1 \mathrm{H}), 9.85(\mathrm{~s}, 1 \mathrm{H})$. HRMS-EI $(\mathrm{m} / \mathrm{z}):[\mathrm{M}]^{+}$calcd for $\mathrm{C}_{26} \mathrm{H}_{27} \mathrm{NOS}, 401.1813$; found, 401.1821 .

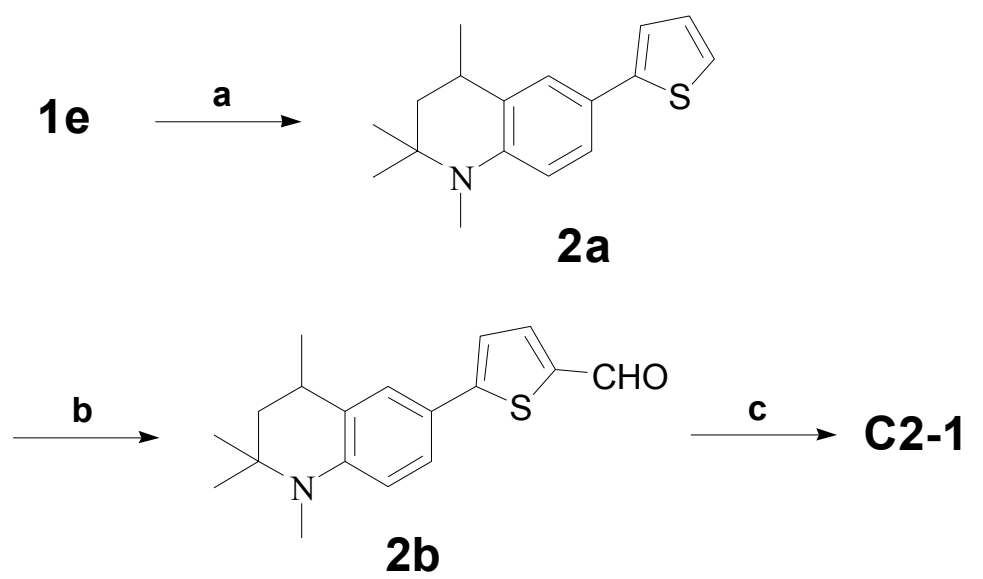

Scheme S3 Synthesis route of C2-1 dye. a) 2-thienylboronic acid, $\mathrm{K}_{2} \mathrm{CO}_{3}, \mathrm{Pd}\left(\mathrm{PPh}_{3}\right)_{4}, \mathrm{DME}$, reflux for 8 h. $85 \%$; b) i. $n$-BuLi, THF, $-15^{\circ} \mathrm{C}, 20 \mathrm{~min}$; ii. DMF, $-15^{\circ} \mathrm{C}, 2 \mathrm{~h}$; iii. water; c) cyano-acetic acid, $\mathrm{CH}_{3} \mathrm{CN}$, piperidine, reflux for $2 \mathrm{~h}$.

\section{1,2,2,4-Tetramethyl-6-thiophen-2-yl-1,2,3,4-tetrahydro-quinoline (2a)}

$\mathrm{Pd}\left(\mathrm{PPh}_{3}\right)_{4}(100 \mathrm{mg}, 0.09 \mathrm{mmol})$ was degassed in vacuo for $30 \mathrm{~min}$ and then 1,2-dimethoxy-ethane (DME, 20mL) was added. 1e (290 mg, $1.08 \mathrm{mmol})$, aqueous $\mathrm{K}_{2} \mathrm{CO}_{3}(1 \mathrm{~mL}, 2 \mathrm{M}, 2 \mathrm{mmol})$ and 2-thienylboronic acid (210 mg, $\left.1.64 \mathrm{mmol}\right)$ were added to the $\mathrm{Pd}\left(\mathrm{PPh}_{3}\right)_{4}$ solution and this mixture was degassed with a steady stream of argon for $20 \mathrm{~min}$ at room temperature. The reaction mixture was then heated to reflux for $8 \mathrm{~h}$ under argon. After cooled to room temperature, the reaction mixture was extracted by $\mathrm{CH}_{2} \mathrm{Cl}_{2}(3 \times 30 \mathrm{~mL})$. The organic layer was collected and evaporated in vacuo, the residue was subjected to a column chromatography (silica gel, 
$\mathrm{CH}_{2} \mathrm{Cl}_{2}$ :hexane $=1: 5$ as eluent $)$ to give the target compound $\mathbf{2 a}$ as yellow oil $(250 \mathrm{mg}$, 85\%). ${ }^{1} \mathrm{H}$ NMR (Acetone-d $\left.6,400 \mathrm{MHz}, \mathrm{ppm}\right): \delta 1.22$ (s, 3H), 1.30 (s, 3H), 1.37 (d, $J=$ $6.6 \mathrm{~Hz}, 3 \mathrm{H}), 1.47\left(\mathrm{dd}, J_{1}=12.8 \mathrm{~Hz}, J_{2}=12.9 \mathrm{~Hz}, 1 \mathrm{H}\right), 1.86\left(\mathrm{dd}, J_{1}=4.3 \mathrm{~Hz}, J_{2}=12.9\right.$ $\mathrm{Hz}, 1 \mathrm{H}), 2.84-2.86(\mathrm{~m}, 4 \mathrm{H}), 6.59(\mathrm{~d}, J=8.5 \mathrm{~Hz}, 1 \mathrm{H}), 7.03\left(\mathrm{dd}, J_{l}=3.9 \mathrm{~Hz}, J_{2}=4.5\right.$ $\mathrm{Hz}, 1 \mathrm{H}), 7.21$ (d, $J=3.0 \mathrm{~Hz}, 1 \mathrm{H}), 7.24$ (d, $J=5.0 \mathrm{~Hz}, 1 \mathrm{H}), 7.33$ (d, $J=8.5 \mathrm{~Hz}, 1 \mathrm{H})$, 7.37 (s, 1H). HRMS-EI $(\mathrm{m} / \mathrm{z})$ : [M] $]^{+}$calcd for $\mathrm{C}_{17} \mathrm{H}_{21} \mathrm{NS}, 271.1395$; found, 271.1391 .

5-(1,2,2,4-Tetramethyl-1,2,3,4-tetrahydro-quinolin-6-yl)-thiophene-2-carbaldehyde (2b). 2b was prepared by the similar procedure of synthesizing 1f. Green solid (yield 72\%). Mp. 90-92 ${ }^{\circ} \mathrm{C} .{ }^{1} \mathrm{H}$ NMR (Acetone- $\left.\mathrm{d}_{6}, 400 \mathrm{MHz}, \mathrm{ppm}\right): \delta 1.25$ (s, 3H), 1.34 (s, $3 \mathrm{H}), 1.40(\mathrm{~d}, J=6.8 \mathrm{~Hz}, 3 \mathrm{H}), 1.49\left(\mathrm{dd}, J_{1}=12.7 \mathrm{~Hz}, J_{2}=13.6 \mathrm{~Hz}, 1 \mathrm{H}\right), 1.89\left(\mathrm{dd}, J_{1}=\right.$ $\left.4.7 \mathrm{~Hz}, J_{2}=13.0 \mathrm{~Hz}, 1 \mathrm{H}\right), 2.87-2.90(\mathrm{~m}, 4 \mathrm{H}), 6.64(\mathrm{~d}, J=8.6 \mathrm{~Hz}, 1 \mathrm{H}), 7.44(\mathrm{~d}, J=$ $3.7 \mathrm{~Hz}, 1 \mathrm{H}), 7.48-7.50(\mathrm{~m}, 2 \mathrm{H}), 7.88$ (d, $J=4.0 \mathrm{~Hz}, 1 \mathrm{H}), 9.85$ (s, 1H). HRMS-EI (m/z): [M] $]^{+}$calcd for $\mathrm{C}_{18} \mathrm{H}_{21} \mathrm{NOS}, 299.1344$; found, 299.1341 .

Bis-formylation reaction for the synthesis of intermediates $\mathbf{5 b - 8 b}$

The general procedure for the synthesis of intermediates $\mathbf{5 b - 8 b}$ was described. For example, the synthesis of compound $\mathbf{7 b}$ was as follows. The compound $7 \mathbf{a}$ (760 $\mathrm{mg}$, $3.88 \mathrm{mmol}$ ) was dried in vacuo for $30 \mathrm{~min}$, and then anhydrous THF $(30 \mathrm{~mL})$ was added. A solution of $n$-BuLi in hexane $(2.57 \mathrm{M}, 3.2 \mathrm{~mL}, 8.2 \mathrm{mmol})$ was added dropwise at $0{ }^{\circ} \mathrm{C}$. After the addition of $n$-BuLi, the solution was stirred at r. t. for $1 \mathrm{~h}$ and then cooled to $-78{ }^{\circ} \mathrm{C}$. After cooling, anhydrous DMF (1.5mL, $\left.19.4 \mathrm{mmol}\right)$ was added dropwise to this solution at $-78{ }^{\circ} \mathrm{C}$. The mixture was stirred at $-78{ }^{\circ} \mathrm{C}$ for another $1 \mathrm{~h}$ and then warmed to r. t. slowly. After stirring at r. t. for $2 \mathrm{~h}$, the mixture was poured into ice water $(200 \mathrm{~mL})$. A lot of yellow solid was precipitated. The precipitate was filtered and dried in vacuo to give the target compound $7 \mathbf{b}$ with enough purity for the next reaction. For other examples, a further purification procedure by chromatography on silica gel $\left(\mathrm{CH}_{2} \mathrm{Cl}_{2}\right.$ as eluent) was needed.

5,5'-Diformyl-2,2'-bithiophene(5b)

Yellow solid (yield 50\%). Mp. 212-213 ${ }^{\circ} \mathrm{C}$ (lit. $\left.185-195 \quad{ }^{\circ} \mathrm{C}\right) .{ }^{[5]}{ }^{1} \mathrm{H} \quad \mathrm{NMR}$ (Acetone-d $\left.\mathrm{d}_{6}, 400 \mathrm{MHz}, \mathrm{ppm}\right): \delta 7.71(\mathrm{~d}, J=3.9 \mathrm{~Hz}, 2 \mathrm{H}), 8.01(\mathrm{~d}, J=3.9 \mathrm{~Hz}, 2 \mathrm{H})$, 9.99 (s, 2H). HRMS-EI $(\mathrm{m} / \mathrm{z})$ : $[\mathrm{M}]^{+}$calcd for $\mathrm{C}_{10} \mathrm{H}_{6} \mathrm{O}_{2} \mathrm{~S}_{2}, 221.9809$; found, 221.9803. 


\section{5,5"'-Diformyl-2,2';5',2"'-terthiophene (6b)}

Red brown solid (yield 76\%). Mp. 202-207 ${ }^{\circ} \mathrm{C}$ (lit. 203-205 $\left.{ }^{\circ} \mathrm{C}\right) .{ }^{[6]}{ }^{1} \mathrm{H} \mathrm{NMR}\left(\mathrm{CDCl}_{3}\right.$, 400MHz, ppm): $\delta 7.30$ (d, $J=4.0 \mathrm{~Hz}, 2 \mathrm{H}), 7.32(\mathrm{~s}, 2 \mathrm{H}), 7.70$ (d, $J=4.0 \mathrm{~Hz}, 2 \mathrm{H}), 9.89$ (s, 2H). HRMS-EI (m/z): [M] $]^{+}$calcd for $\mathrm{C}_{14} \mathrm{H}_{8} \mathrm{O}_{2} \mathrm{~S}_{3}, 303.9686$; found, 303.9688 .

2,6-Diformyl-dithieno[3,2-b;2',3'-d]thiophene (7b)

Yellow solid (yield 94\%). Mp. $>260{ }^{\circ} \mathrm{C}$ (lit. $270{ }^{\circ} \mathrm{C}$, decomposition). ${ }^{[7]}{ }^{1} \mathrm{H}$ NMR (Acetone- $\left.\mathrm{d}_{6}, 400 \mathrm{MHz}, \mathrm{ppm}\right): \delta 8.48(\mathrm{~s}, 2 \mathrm{H}), 10.11(\mathrm{~s}, 2 \mathrm{H}) . \operatorname{HRMS}-\mathrm{EI}(\mathrm{m} / \mathrm{z}):[\mathrm{M}]^{+}$ calcd for $\mathrm{C}_{10} \mathrm{H}_{4} \mathrm{O}_{2} \mathrm{~S}_{3}, 251.9373$; found, 251.9373 .

E-1,2-Bis(2-formyl-5-thienyl)ethene (8b)

Brown solid (yield 48\%). Mp. 197-205 ${ }^{\circ} \mathrm{C}$ (lit. 203-205 $\left.{ }^{\circ} \mathrm{C}\right) .{ }^{[8]}{ }^{1} \mathrm{H}$ NMR (Acetone- $\left.\mathrm{d}_{6}, 400 \mathrm{MHz}, \mathrm{ppm}\right): \delta 7.49$ (d, $\left.J=3.9 \mathrm{~Hz}, 2 \mathrm{H}\right), 7.54$ (s, 2H), 7.93 (d, $J=3.9$ $\mathrm{Hz}, 2 \mathrm{H}), 9.95(\mathrm{~s}, 2 \mathrm{H})$. HRMS-EI $(\mathrm{m} / \mathrm{z}):[\mathrm{M}]^{+}$calcd for $\mathrm{C}_{12} \mathrm{H}_{8} \mathrm{O}_{2} \mathrm{~S}_{2}, 247.9966$; found, 247.9963.

Mono-Witting reaction for the synthesis of intermediates $\mathbf{5 c - 8 c}$

A typical preparation of the intermediates $\mathbf{5 c - 8 c}$ was as follows for the particular case of the terthiophene spacer 6c. The precursor dicarbaldehyde $6 \mathbf{b}$ (304 mg, 1 $\mathrm{mmol}$ ), anhydrous $\mathrm{K}_{2} \mathrm{CO}_{3}(276 \mathrm{mg}, 2 \mathrm{mmol})$ and 18-crown-6-ether (15mg) were dried in vacuo for $30 \mathrm{~min}$, and then added $40 \mathrm{~mL}$ of dry DMF. Ylide $\mathbf{1 h}(544 \mathrm{mg}, 1 \mathrm{mmol})$ was dissolved in dry DMF $(10 \mathrm{~mL})$ and added dropwise to the above solution slowly. After the addition the mixture was stirred at $\mathrm{r}$. $\mathrm{t}$. for another $2 \mathrm{~h}$. The mixture was poured into ice water $(200 \mathrm{~mL})$ and leading to precipitate red solid. The precipitate was filtered and purified by chromatography on silica gel with $\mathrm{CH}_{2} \mathrm{Cl}_{2}$-hexane (1:1) as eluent. The final compound $\mathbf{6 c}$ was obtained as orange crystals.

E-1-[1,2,2,4-Tetramethyl-1,2,3,4-tetrahydro-quinolin-6-yl]-2-[5-formyl-2,2'-bithiophe ne-5'-yl]ethane(5c). Red brown solid (yield 56\%). Mp. 148-150 ${ }^{\circ} \mathrm{C} .{ }^{1} \mathrm{H}$ NMR (Acetone- $\left.\mathrm{d}_{6}, 400 \mathrm{MHz}, \mathrm{ppm}\right): \delta 1.23(\mathrm{~s}, 3 \mathrm{H}), 1.31(\mathrm{~s}, 3 \mathrm{H}), 1.37(\mathrm{~d}, J=6.7 \mathrm{~Hz}, 3 \mathrm{H})$, $1.47\left(\mathrm{dd}, J_{l}=12.7 \mathrm{~Hz}, J_{2}=13.0 \mathrm{~Hz}, 1 \mathrm{H}\right), 1.87\left(\mathrm{dd}, J_{l}=4.4 \mathrm{~Hz}, J_{2}=13.0 \mathrm{~Hz}, 1 \mathrm{H}\right)$, 2.85-2.86 (m, 4H), 6.59 (d, $J=8.6 \mathrm{~Hz}, 1 \mathrm{H}), 6.95(\mathrm{~d}, J=16.0 \mathrm{~Hz}, 1 \mathrm{H}), 7.08(\mathrm{~d}, J=3.7$ $\mathrm{Hz}, 1 \mathrm{H}), 7.17$ (d, $J=16.0 \mathrm{~Hz}, 1 \mathrm{H}), 7.29$ (d, $J=8.5 \mathrm{~Hz}, 1 \mathrm{H}), 7.35$ (s, 1H), 7.42-7.45 $(\mathrm{m}, 2 \mathrm{H}), 7.92(\mathrm{~d}, J=3.8 \mathrm{~Hz}, 1 \mathrm{H}), 9.91(\mathrm{~s}, 1 \mathrm{H})$. HRMS-EI $(\mathrm{m} / \mathrm{z}):[\mathrm{M}]^{+}$calcd for 
$\mathrm{C}_{24} \mathrm{H}_{25} \mathrm{NOS}_{2}$, 407.1378; found, 407.1372.

E-1-[1,2,2,4-Tetramethyl-1,2,3,4-tetrahydro-quinolin-6-yl]-2-[5-formyl-2,2';5',2”-t erthiophene-5"'-yl]ethane(6c). Red brown solid (yield 42\%). Mp. 138-140 ${ }^{\circ} \mathrm{C} .{ }^{1} \mathrm{H}$ NMR (Acetone- $\left.\mathrm{d}_{6}, 400 \mathrm{MHz}, \mathrm{ppm}\right): \delta 1.23(\mathrm{~s}, 3 \mathrm{H}), 1.31$ (s, 3H), 1.38 (d, $J=6.7 \mathrm{~Hz}$, $3 \mathrm{H}), 1.47\left(\mathrm{dd}, J_{1}=12.7 \mathrm{~Hz}, J_{2}=13.8 \mathrm{~Hz}, 1 \mathrm{H}\right), 1.87\left(\mathrm{dd}, J_{1}=4.0 \mathrm{~Hz}, J_{2}=13.8 \mathrm{~Hz}\right.$, 1H), 2.85-2.86 (m, 4H), $6.58(\mathrm{~d}, J=8.9 \mathrm{~Hz}, 1 \mathrm{H}), 6.93(\mathrm{~d}, J=16.2 \mathrm{~Hz}, 1 \mathrm{H}), 7.03$ (d, $J$ $=3.9 \mathrm{~Hz}, 1 \mathrm{H}), 7.15(\mathrm{~d}, J=16.3 \mathrm{~Hz}, 1 \mathrm{H}), 7.28-7.30(\mathrm{~m}, 3 \mathrm{H}), 7.34(\mathrm{~s}, 1 \mathrm{H}), 7.49(\mathrm{~d}, J=$ $4.1 \mathrm{~Hz}, 1 \mathrm{H}), 7.51(\mathrm{~d}, J=4.1 \mathrm{~Hz}, 1 \mathrm{H}), 7.95(\mathrm{~d}, J=3.9 \mathrm{~Hz}, 1 \mathrm{H}), 9.93(\mathrm{~s}, 1 \mathrm{H})$. HRMS-EI $(\mathrm{m} / \mathrm{z})$ : [M] ${ }^{+}$calcd for $\mathrm{C}_{28} \mathrm{H}_{27} \mathrm{NOS}_{3}, 489.1255$; found, 489.1255.

E-1-[1,2,2,4-Tetramethyl-1,2,3,4-tetrahydro-quinolin-6-yl]-2-[6-formyl-dithieno[3, 2-b;2',3'-d]thiophene-2-yl]ethane(7c). Orange red solid (yield 60\%). Mp. 185-190 ${ }^{\circ} \mathrm{C}$. ${ }^{1} \mathrm{H}$ NMR (Acetone- $\left.\mathrm{d}_{6}, 400 \mathrm{MHz}, \mathrm{ppm}\right): \delta 1.24(\mathrm{~s}, 3 \mathrm{H}), 1.32(\mathrm{~s}, 3 \mathrm{H}), 1.38(\mathrm{~d}, J=6.6$ $\mathrm{Hz}, 3 \mathrm{H}), 1.48\left(\mathrm{dd}, J_{l}=12.9 \mathrm{~Hz}, J_{2}=13.3 \mathrm{~Hz}, 1 \mathrm{H}\right), 1.87\left(\mathrm{dd}, J_{l}=4.4 \mathrm{~Hz}, J_{2}=12.9 \mathrm{~Hz}\right.$, 1H), 2.86-2.87 (m, 4H), $6.60(\mathrm{~d}, J=8.9 \mathrm{~Hz}, 1 \mathrm{H}), 7.06(\mathrm{~d}, J=16.1 \mathrm{~Hz}, 1 \mathrm{H}), 7.25-7.33$ (m, 2H), $7.35(\mathrm{~s}, 1 \mathrm{H}), 7.47(\mathrm{~s}, 1 \mathrm{H}), 8.35(\mathrm{~s}, 1 \mathrm{H}), 10.01(\mathrm{~s}, 1 \mathrm{H})$. HRMS-EI $(\mathrm{m} / \mathrm{z}):[\mathrm{M}]^{+}$ calcd for $\mathrm{C}_{24} \mathrm{H}_{23} \mathrm{NOS}_{3}, 437.0942$; found, 437.0948 .

E-1-[2-E-(1,2,2,4-Tetramethyl-1,2,3,4-tetrahydro-quinolin-6-yl)-5-thienyl]-2-[2-for myl-5-thienyl]ethane (8c). Red brown solid (yield 54\%). Mp. 115-118 ${ }^{\circ} \mathrm{C} .{ }^{1} \mathrm{H}$ NMR (Acetone- $\left.\mathrm{d}_{6}, 400 \mathrm{MHz}, \mathrm{ppm}\right): \delta 1.23(\mathrm{~s}, 3 \mathrm{H}), 1.31(\mathrm{~s}, 3 \mathrm{H}), 1.37(\mathrm{~d}, J=6.6 \mathrm{~Hz}, 3 \mathrm{H})$, $1.47\left(\mathrm{dd}, J_{1}=12.8 \mathrm{~Hz}, J_{2}=13.2 \mathrm{~Hz}, 1 \mathrm{H}\right), 1.86\left(\mathrm{dd}, J_{1}=4.5 \mathrm{~Hz}, J_{2}=13.0 \mathrm{~Hz}, 1 \mathrm{H}\right)$, 2.84-2.86 (m, 4H), $6.58(\mathrm{~d}, J=8.7 \mathrm{~Hz}, 1 \mathrm{H}), 6.93(\mathrm{~d}, J=16.0 \mathrm{~Hz}, 1 \mathrm{H}), 7.00(\mathrm{~d}, J=3.7$ $\mathrm{Hz}, 1 \mathrm{H}), 7.11(\mathrm{~d}, J=15.7 \mathrm{~Hz}, 1 \mathrm{H}), 7.18(\mathrm{~d}, J=3.8 \mathrm{~Hz}, 1 \mathrm{H}), 7.26\left(\mathrm{dd}, J_{l}=2.0 \mathrm{~Hz}, J_{2}\right.$ $=8.6 \mathrm{~Hz}, 1 \mathrm{H}), 7.33-7.34(\mathrm{~m}, 2 \mathrm{H}), 7.42(\mathrm{~d}, J=15.9 \mathrm{~Hz}, 1 \mathrm{H}), 7.87(\mathrm{~d}, J=3.9 \mathrm{~Hz}, 1 \mathrm{H})$, 9.89 (s, 1H). HRMS-EI $(m / z)$ : $[\mathrm{M}]^{+}$calcd for $\mathrm{C}_{26} \mathrm{H}_{27} \mathrm{NOS}_{2}, 433.1534$; found, 433.1539 . 

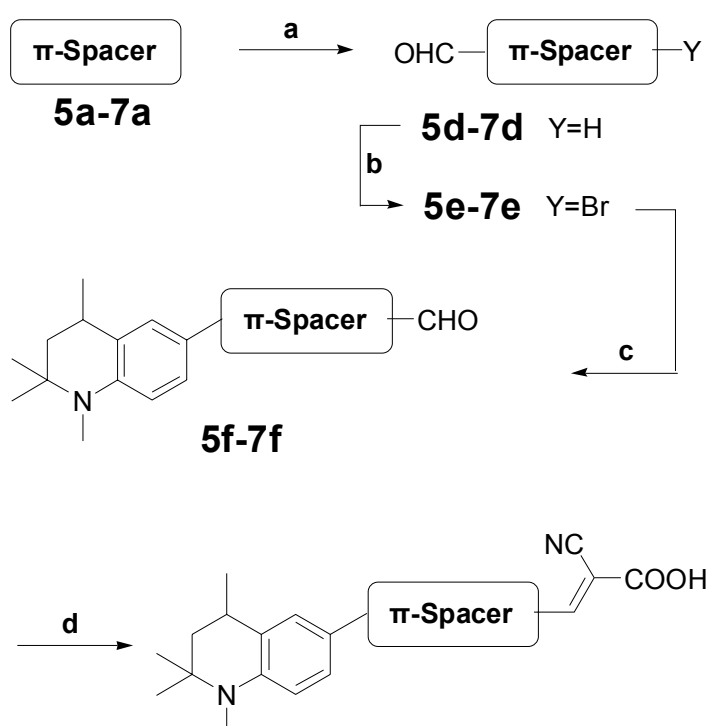

\section{$C 2-2-C 2-4$}

Scheme S3 Synthesis route of C2-2 - C2-4 dyes. a) $\mathrm{POCl}_{3} / \mathrm{DMF}, \mathrm{CH}_{2} \mathrm{ClCH}_{2} \mathrm{Cl}$, reflux, 4h; b) NBS, DMF, r. t. overnight; c) 1f, $\mathrm{Pd}\left(\mathrm{PPh}_{3}\right)_{4}, \mathrm{~K}_{2} \mathrm{CO}_{3}$, DME, reflux, overnight; d) cyano-acetic acid, $\mathrm{CH} 3 \mathrm{CN}$, reflux, $2 \mathrm{~h}$.

Mono-formylation reaction for the synthesis of intermidates $\mathbf{5 d - 7 d}$

As a representative example, a typical preparation of the intermediates $\mathbf{5 d}$ was as follows. To a solution of compound $5 \mathbf{a}(417 \mathrm{mg}, 2.5 \mathrm{mmol})$ and anhydrous DMF $(0.25 \mathrm{~mL}, 3.2 \mathrm{mmol})$ in anhydrous 1,2-dichloroethane $(5 \mathrm{~mL})$ was added $\mathrm{POCl}_{3}$ $(0.24 \mathrm{~mL}, 2.6 \mathrm{mmol})$, and the mixture was refluxed for $4 \mathrm{~h}$. After cooling, the mixture was poured into ice water $(50 \mathrm{~mL})$ and stirred for $2 \mathrm{~h}$. After separation of the organic phase by decantation, the aqueous phase was extracted with $\mathrm{CH}_{2} \mathrm{Cl}_{2}(3 \times 30 \mathrm{~mL})$. The organic fractions were gathered, dried over $\mathrm{MgSO}_{4}$, and evaporated in vacuo. After chromatography on silica gel $\left(\mathrm{CH}_{2} \mathrm{Cl}_{2}\right.$ as elute), yellow solid was obtained.

5-Formyl-2,2'-bithiophene (5d)

Yellow green solid (yield 68\%). Mp. 55-56 ${ }^{\circ} \mathrm{C}$ (lit. 57-58 ${ }^{\circ} \mathrm{C}$ ). ${ }^{[9]}{ }^{1} \mathrm{H}$ NMR $\left(\mathrm{CDCl}_{3}\right.$, 400MHz, ppm): $\delta 7.08\left(\mathrm{dd}, J_{1}=4.3 \mathrm{~Hz}, J_{2}=4.4 \mathrm{~Hz}, 1 \mathrm{H}\right), 7.26(\mathrm{~d}, J=3.8 \mathrm{~Hz}, 1 \mathrm{H})$, 7.36-7.37 (m, 2H), $7.68(\mathrm{~d}, J=3.9 \mathrm{~Hz}, 1 \mathrm{H}), 9.87(\mathrm{~s}, 1 \mathrm{H})$. HRMS-EI $(\mathrm{m} / \mathrm{z}):[\mathrm{M}]^{+}$calcd for $\mathrm{C}_{9} \mathrm{H}_{6} \mathrm{OS}_{2}, 193.9860$; found, 193.9854 .

5-Formyl-2,2';5',2'"-terthiophene (6d)

Orange solid (yield 65\%). Mp. 132-133 ${ }^{\circ} \mathrm{C}$ (lit. 140-141 ${ }^{\circ} \mathrm{C}$ ). ${ }^{[10]}{ }^{1} \mathrm{H}-\mathrm{NMR}$ (Acetone-d $6,400 \mathrm{MHz}, \mathrm{ppm}): \delta 7.13\left(\mathrm{dd}, J_{1}=3.9 \mathrm{~Hz}, J_{2}=4.9 \mathrm{~Hz}, 1 \mathrm{H}\right), 7.32(\mathrm{~d}, J=$ 
$3.9 \mathrm{~Hz}, 1 \mathrm{H}), 7.40(\mathrm{~d}, J=3.4 \mathrm{~Hz}, 1 \mathrm{H}), 7.51(\mathrm{~m}, 3 \mathrm{H}), 7.95$ (d, $J=3.9 \mathrm{~Hz}, 1 \mathrm{H}), 9.94$ (s, 1H). HRMS-EI $(\mathrm{m} / \mathrm{z}):[\mathrm{M}]^{+}$calcd for $\mathrm{C}_{19} \mathrm{H}_{8} \mathrm{OS}_{3}, 295.9737$; found, 295.9725.

2-Formyl-dithieno[3,2-b;2',3'-d]thiophene (7d)

White solid (yield 78\%). Mp. 159-160 ${ }^{\circ} \mathrm{C}$ (lit. 161-162 ${ }^{\circ} \mathrm{C}$ ). ${ }^{[11]}{ }^{1} \mathrm{H}$ NMR (Acetone- $\left.\mathrm{d}_{6}, 400 \mathrm{MHz}, \mathrm{ppm}\right): \delta 7.59$ (d, $\left.J=5.3 \mathrm{~Hz}, 1 \mathrm{H}\right), 7.87$ (d, $\left.J=5.3 \mathrm{~Hz}, 1 \mathrm{H}\right)$, $8.39(\mathrm{~s}, 1 \mathrm{H}), 10.04(\mathrm{~s}, 1 \mathrm{H})$. HRMS-EI $(\mathrm{m} / \mathrm{z})$ : $[\mathrm{M}]^{+}$calcd for $\mathrm{C}_{9} \mathrm{H}_{4} \mathrm{OS}_{3}, 223.9424$; found, 223.9416 .

NBS bromization reaction for the synthesis of intermidates $\mathbf{5 e - 7 e}$

A typical procedure of synthesis of compound 6e was as follows. 6d (210 mg, 0.76 mmol) and NBS (149mg, 0.84mmol) were dissolved in DMF (20mL) and stirred at r. t. for $24 \mathrm{~h}$. The mixture was poured into water $(100 \mathrm{~mL})$ and leading to precipitate red solid. The precipitate was filtered and purified by chromatography on silica gel $\left(\mathrm{CH}_{2} \mathrm{Cl}_{2}\right.$ as eluent) to give the compound $\mathbf{6 e}$ as orange solid.

5-Bromo-5'-fromyl-2,2'-bithiophene (5e)

Yellow solid (yield 96\%). Mp. 139-140 ${ }^{\circ} \mathrm{C}$ (lit. $145{ }^{\circ} \mathrm{C}$ ). ${ }^{[12]}{ }^{1} \mathrm{H}$ NMR (Acetone-d $\mathrm{d}_{6}$, 400MHz, ppm): $\delta 7.25$ (d, $J=3.8 \mathrm{~Hz}, 1 \mathrm{H}), 7.38$ (d, $J=3.9 \mathrm{~Hz}, 1 \mathrm{H}), 7.47$ (d, $J=3.9$ $\mathrm{Hz}, 1 \mathrm{H}), 7.94(\mathrm{~d}, J=3.9 \mathrm{~Hz}, 1 \mathrm{H}), 9.94(\mathrm{~s}, 1 \mathrm{H})$. HRMS-EI $(\mathrm{m} / \mathrm{z}):[\mathrm{M}]^{+}$calcd for $\mathrm{C}_{9} \mathrm{H}_{5} \mathrm{BrOS}_{2}, 271.8965$; found, 271.8967; 273.8947.

\section{5-Bromo-5"'-formyl-2,2';5',2"'-terthiophene (6e)}

Orange solid (yield 96\%). Mp. 150-152 ${ }^{\circ} \mathrm{C}$ (lit. $158{ }^{\circ} \mathrm{C}$ ). ${ }^{[13]}{ }^{1} \mathrm{H}$ NMR (Acetone- $\mathrm{d}_{6}$, 400MHz, ppm): $\delta 7.20$ (d, $J=3.9 \mathrm{~Hz}, 1 \mathrm{H}), 7.22(\mathrm{~d}, J=3.9 \mathrm{~Hz}, 1 \mathrm{H}), 7.32(\mathrm{~d}, J=3.9$ $\mathrm{Hz}, 1 \mathrm{H}), 7.51-7.52(\mathrm{~m}, 2 \mathrm{H}), 7.95(\mathrm{~d}, J=4.0 \mathrm{~Hz}, 1 \mathrm{H}), 9.94(\mathrm{~s}, 1 \mathrm{H})$. HRMS-EI $(\mathrm{m} / \mathrm{z})$ : $[\mathrm{M}]^{+}$calcd for $\mathrm{C}_{13} \mathrm{H}_{7} \mathrm{BrOS}_{3}, 353.8842$; found, 353.8838; 355.8810 .

2-Bromo-6-formyl- dithieno[3,2-b;2',3'-d]thiophene (7e)

Yellow solid (yield 99\%). Mp. $191{ }^{\circ} \mathrm{C} .{ }^{1} \mathrm{H}$ NMR (Acetone-d 6 , 400MHz, ppm): $\delta$ $7.74(\mathrm{~s}, 1 \mathrm{H}), 8.40(\mathrm{~s}, 1 \mathrm{H}), 10.04(\mathrm{~s}, 1 \mathrm{H})$. HRMS-EI $(\mathrm{m} / \mathrm{z}):[\mathrm{M}]^{+}$calcd for $\mathrm{C}_{9} \mathrm{H}_{3} \mathrm{BrOS}_{3}$, 301.8529; found, 301.8524; 303.8500 .

Suzuki coupling reaction for the synthesis of intermediates $\mathbf{5 f - 7 f}$

A typical preparation of the intermediates $\mathbf{5 f - 7 f}$ was as follows for the particular case of the compound 6f. $\mathrm{Pd}\left(\mathrm{PPh}_{3}\right)_{4}(80 \mathrm{mg}, 0.07 \mathrm{mmol})$ was degassed in vacuo for 30 
min and then 1,2-dimethoxy-ethane (DME, 25mL) was added. The bromated intermediate 6e $(178 \mathrm{mg}, 0.5 \mathrm{mmol})$, aqueous $\mathrm{K}_{2} \mathrm{CO}_{3}(1 \mathrm{~mL}, 2 \mathrm{M}, 2 \mathrm{mmol})$ and $\mathbf{1 f}$ (140mg, 0.6mmol) were added to the $\mathrm{Pd}\left(\mathrm{PPh}_{3}\right)_{4}$ solution and this mixture was degassed with a steady stream of argon for $20 \mathrm{~min}$ at room temperature. The reaction mixture was then heated to reflux overnight $(\sim 15 \mathrm{~h})$ under argon. After cooled to room temperature, the reaction mixture was extracted by $\mathrm{CH}_{2} \mathrm{Cl}_{2}(3 \times 30 \mathrm{~mL})$. The organic layer was collected and evaporated in vacuo, the residue was subjected to a column chromatography (silica gel, $\mathrm{CH}_{2} \mathrm{Cl}_{2}$ :hexane $=1: 1$ as eluent) to give the target compound $\mathbf{6 f}$ as red-orange solid.

5'-(1,2,2,4-Tetramethyl-1,2,3,4-tetrahydro-quinolin-6-yl)-[2,2']bithiophenyl-5-carbald ehyde (5f)

Red orange solid (yield 63\%). Mp. 117-119 ${ }^{\circ} \mathrm{C} .{ }^{1} \mathrm{H}$ NMR (Acetone-d 6 , 400MHz, ppm): $\delta 1.24(\mathrm{~s}, 3 \mathrm{H}), 1.33(\mathrm{~s}, 3 \mathrm{H}), 1.40(\mathrm{~d}, J=6.7 \mathrm{~Hz}, 3 \mathrm{H}), 1.49\left(\mathrm{dd}, J_{l}=12.6 \mathrm{~Hz}, J_{2}\right.$ $=12.8 \mathrm{~Hz}, 1 \mathrm{H}), 1.89\left(\mathrm{dd}, J_{l}=4.5 \mathrm{~Hz}, J_{2}=13.1 \mathrm{~Hz}, 1 \mathrm{H}\right), 2.88-2.89(\mathrm{~m}, 4 \mathrm{H}), 6.63(\mathrm{~d}, J$ $=8.4 \mathrm{~Hz}, 1 \mathrm{H}), 7.29(\mathrm{~d}, J=3.9 \mathrm{~Hz}, 1 \mathrm{H}), 7.39-7.44(\mathrm{~m}, 3 \mathrm{H}), 7.48(\mathrm{~d}, J=3.9 \mathrm{~Hz}, 1 \mathrm{H})$, $7.92(\mathrm{~d}, J=4.0 \mathrm{~Hz}, 1 \mathrm{H}), 9.91(\mathrm{~s}, 1 \mathrm{H})$. HRMS-EI $(\mathrm{m} / \mathrm{z}):[\mathrm{M}]^{+}$calcd for $\mathrm{C}_{22} \mathrm{H}_{23} \mathrm{NOS}_{2}$, 381.1221; found, 381.1228 .

5"-(1,2,2,4-Tetramethyl-1,2,3,4-tetrahydro-quinolin-6-yl)-[2,2';5',2"]terthiophene-5-ca rbaldehyde (6f)

Red-orange solid (yield 80 \%). Mp.: 140-145 ${ }^{\circ} \mathrm{C} .{ }^{1} \mathrm{H}$ NMR (Acetone-d 6 , 400MHz, ppm): $\delta 1.24(\mathrm{~s}, 3 \mathrm{H}), 1.32(\mathrm{~s}, 3 \mathrm{H}), 1.39(\mathrm{~d}, J=6.7 \mathrm{~Hz}, 3 \mathrm{H}), 1.49\left(\mathrm{dd}, J_{l}=12.1 \mathrm{~Hz}, J_{2}\right.$ $=13.4 \mathrm{~Hz}, 1 \mathrm{H}), 1.89\left(\mathrm{dd}, J_{l}=4.3 \mathrm{~Hz}, J_{2}=12.3 \mathrm{~Hz}, 1 \mathrm{H}\right), 2.86-2.87(\mathrm{~m}, 4 \mathrm{H}), 6.63(\mathrm{~d}, J$ $=8.6 \mathrm{~Hz}, 1 \mathrm{H}), 7.24(\mathrm{~d}, J=3.8 \mathrm{~Hz}, 1 \mathrm{H}), 7.29(\mathrm{~d}, J=3.9 \mathrm{~Hz}, 1 \mathrm{H}), 7.32(\mathrm{~d}, J=3.7 \mathrm{~Hz}$, 1H), $7.38(\mathrm{~d}, J=8.6 \mathrm{~Hz}, 1 \mathrm{H}), 7.41(\mathrm{~s}, 1 \mathrm{H}), 7.48(\mathrm{~d}, J=4.0 \mathrm{~Hz}, 1 \mathrm{H}), 7.51(\mathrm{~d}, J=3.9$ $\mathrm{Hz}, 1 \mathrm{H}), 7.94(\mathrm{~d}, J=4.0 \mathrm{~Hz}, 1 \mathrm{H}), 9.93$ (s, 1H). HRMS-EI $(m / z):[\mathrm{M}]^{+}$calcd for $\mathrm{C}_{26} \mathrm{H}_{25} \mathrm{NOS}_{3}$, 463.1098; found, 463.1090 .

6-(1,2,2,4-Tetramethyl-1,2,3,4-tetrahydro-quinolin-6-yl)-dithieno[3,2-b;2',3'-d]thioph ene-2-carbaldehyde (7f)

Red-orange solid (yield $44 \%$ ). Mp.: 183-184 ${ }^{\circ} \mathrm{C} .{ }^{1} \mathrm{H}$ NMR (Acetone-d 6 , 400MHz, ppm): $\delta 1.26(\mathrm{~s}, 3 \mathrm{H}), 1.34(\mathrm{~s}, 3 \mathrm{H}), 1.42(\mathrm{~d}, J=6.6 \mathrm{~Hz}, 3 \mathrm{H}), 1.51\left(\mathrm{dd}, J_{l}=12.7 \mathrm{~Hz}, J_{2}\right.$ 
$=13.2 \mathrm{~Hz}, 1 \mathrm{H}), 1.91\left(\mathrm{dd}, J_{1}=5.0 \mathrm{~Hz}, J_{2}=12.4 \mathrm{~Hz}, 1 \mathrm{H}\right), 2.90(\mathrm{~s}, 3 \mathrm{H}), 2.92(\mathrm{~m}, 1 \mathrm{H})$, $6.66(\mathrm{~d}, J=8.8 \mathrm{~Hz}, 1 \mathrm{H}), 7.46(\mathrm{~d}, J=8.8 \mathrm{~Hz}, 1 \mathrm{H}), 7.49$ (s, 1H), 7.69 (s, 1H), 8.35 (s, $1 \mathrm{H}), 10.01(\mathrm{~s}, 1 \mathrm{H})$. HRMS-EI $(\mathrm{m} / \mathrm{z})$ : $[\mathrm{M}]^{+}$calcd for $\mathrm{C}_{22} \mathrm{H}_{21} \mathrm{NOS}_{3}, 411.0785$; found, 411.0781 .

Knoevenagel condensation reaction for the synthesis of $\mathbf{C 1}$ series, $\mathbf{C 2}$ series and $\mathbf{C 3 - 1}$ sensitizers

The $\mathbf{C} 1$ series, $\mathbf{C} 2$ series and C3-1 sensitizers were synthesized under Knoevenagel condensation conditions. Cyanoacetic acid and corresponding aldehyde intermediates were refluxed in acetonitrile under the presence of piperidine as catalyst. After chromatography purfication procedure (silica gel, $\mathrm{CH}_{2} \mathrm{Cl}_{2}: \mathrm{MeOH}=10: 1$ as eluent), the target compounds were obtained as dark or red-brown solids.

2-Cyano-3-\{5-[2-(1,2,2,4-tetramethyl-1,2,3,4-tetrahydro-quinolin-6-yl)-vinyl]-thio phen-2-yl $\}$-acrylic acid (C1-1). Black solid (yield 80\%). Mp. 208-210 ${ }^{\circ} \mathrm{C} .{ }^{1} \mathrm{H}-\mathrm{NMR}$ (DMSO-d 6 , 400MHz, ppm): $\delta 1.17$ (s, 3H), 1.27 (s, 3H), 1.33 (d, $J=6.6 \mathrm{~Hz}, 3 \mathrm{H})$, $1.41\left(\mathrm{dd}, J_{1}=12.6 \mathrm{~Hz}, J_{2}=12.9 \mathrm{~Hz}, 1 \mathrm{H}\right), 1.83\left(\mathrm{dd}, J_{1}=4.2 \mathrm{~Hz}, J_{2}=13.0 \mathrm{~Hz}, 1 \mathrm{H}\right)$, 2.77-2.80 (m, 4H), 6.54 (d, $J=8.7 \mathrm{~Hz}, 1 \mathrm{H}), 7.00$ (d, $J=16.1 \mathrm{~Hz}, 1 \mathrm{H}), 7.18$ (d, $J=3.9$ Hz, 1H), 7.21 (d, $J=16.0 \mathrm{~Hz}, 1 \mathrm{H}), 7.30$ (d, $J=8.5 \mathrm{~Hz}, 1 \mathrm{H}), 7.34$ (s, 1H), 7.59 (d, $J=$ $3.9 \mathrm{~Hz}, 1 \mathrm{H}), 8.06(\mathrm{~s}, 1 \mathrm{H})$. HRMS-EI $(\mathrm{m} / \mathrm{z})$ : $\left[\mathrm{M}-\mathrm{CO}_{2}\right]^{+}$calcd for $\mathrm{C}_{22} \mathrm{H}_{24} \mathrm{~N}_{2} \mathrm{~S}, 348.1660$; found, 348.1656 .

2-Cyano-3-\{5'-[2-(1,2,2,4-tetramethyl-1,2,3,4-tetrahydro-quinolin-6-yl)-vinyl]-[2,2' ]bithiophenyl-5-yl\}-acrylic acid (C1-2). Black solid (yield 65\%). Mp. 202-206 ${ }^{\circ} \mathrm{C}$. ${ }^{1} \mathrm{H}-\mathrm{NMR}\left(\mathrm{DMSO}_{\mathrm{d}}, 400 \mathrm{MHz}, \mathrm{ppm}\right): \delta 1.16$ (s, 3H), 1.26 (s, 3H), 1.33 (d, $J=6.4 \mathrm{~Hz}$, $3 \mathrm{H}), 1.40\left(\mathrm{dd}, J_{1}=12.7 \mathrm{~Hz}, J_{2}=13.5 \mathrm{~Hz}, 1 \mathrm{H}\right), 1.83\left(\mathrm{dd}, J_{1}=4.0 \mathrm{~Hz}, J_{2}=13.3 \mathrm{~Hz}\right.$, 1H), $2.78(\mathrm{~m}, 4 \mathrm{H}), 6.53(\mathrm{~d}, J=8.6 \mathrm{~Hz}, 1 \mathrm{H}), 6.96(\mathrm{~d}, J=16.4 \mathrm{~Hz}, 1 \mathrm{H}), 7.12-7.19(\mathrm{~m}$, 2H), $7.26(\mathrm{~d}, J=8.5 \mathrm{~Hz}, 1 \mathrm{H}), 7.30(\mathrm{~s}, 1 \mathrm{H}), 7.53(\mathrm{~m}, 2 \mathrm{H}), 7.95(\mathrm{~d}, J=3.7 \mathrm{~Hz}, 1 \mathrm{H})$, $8.46(\mathrm{~s}, 1 \mathrm{H})$. HRMS-EI $(\mathrm{m} / \mathrm{z})$ : $\left[\mathrm{M}-\mathrm{CO}_{2}\right]^{+}$calcd for $\mathrm{C}_{26} \mathrm{H}_{26} \mathrm{~N}_{2} \mathrm{~S}_{2}, 430.1538$; found, 430.1532 .

2-Cyano-3-\{5"-[2-(1,2,2,4-tetramethyl-1,2,3,4-tetrahydro-quinolin-6-yl)-vinyl]-[2,2 ';5',2"]terthiophen-5-yl\}-acrylic acid (C1-3). Black solid (yield 72\%). Mp. 215-219 ${ }^{\circ} \mathrm{C}$. ${ }^{1} \mathrm{H}-\mathrm{NMR}$ (DMSO-d 6 , 400MHz, ppm): $\delta 1.17$ (s, 3H), 1.26 (s, 3H), 1.33 (d, $J=6.4 \mathrm{~Hz}$, 
$3 \mathrm{H}), 1.41\left(\mathrm{dd}, J_{l}=12.6 \mathrm{~Hz}, J_{2}=13.4 \mathrm{~Hz}, 1 \mathrm{H}\right), 1.83\left(\mathrm{dd}, J_{1}=4.9 \mathrm{~Hz}, J_{2}=12.8 \mathrm{~Hz}\right.$, 1H), $2.79(\mathrm{~m}, 4 \mathrm{H}), 6.53(\mathrm{~d}, J=8.8 \mathrm{~Hz}, 1 \mathrm{H}), 6.86(\mathrm{~d}, J=15.9 \mathrm{~Hz}, 1 \mathrm{H}), 7.08(\mathrm{~d}, J=3.8$ Hz, 1H), 7.15 (d, $J=16.1 \mathrm{~Hz}, 1 \mathrm{H}), 7.25$ (d, $J=9.0 \mathrm{~Hz}, 1 \mathrm{H}), 7.30$ (s, 1H), 7.36 (m, 2H), $7.59(\mathrm{~d}, J=3.7 \mathrm{~Hz}, 1 \mathrm{H}), 7.97(\mathrm{~d}, J=3.8 \mathrm{~Hz}, 1 \mathrm{H}), 8.46$ (s, 1H). HRMS-EI $(\mathrm{m} / \mathrm{z})$ : $\left[\mathrm{M}-\mathrm{CO}_{2}\right]^{+}$calcd for $\mathrm{C}_{30} \mathrm{H}_{28} \mathrm{~N}_{2} \mathrm{~S}_{3}, 512.1415$; found, 512.1414 .

2-Cyano-3-\{5"-[2-(1,2,2,4-tetramethyl-1,2,3,4-tetrahydro-quinolin-6-yl)-vinyl]-dith ieno[3,2-b;2',3'-d]thiophene-5-yl\}-acrylic acid (C1-4). Dark brown solid (yield 77\%). Mp. $>260{ }^{\circ} \mathrm{C} . \quad{ }^{1} \mathrm{H}-\mathrm{NMR}\left(\mathrm{DMSO}_{\mathrm{d}}, 400 \mathrm{MHz}, \mathrm{ppm}\right): \delta 1.18(\mathrm{~s}, 3 \mathrm{H}), 1.27(\mathrm{~s}, 3 \mathrm{H})$, $1.35(\mathrm{~d}, J=6.7 \mathrm{~Hz}, 3 \mathrm{H}), 1.42\left(\mathrm{dd}, J_{l}=12.7 \mathrm{~Hz}, J_{2}=13.0 \mathrm{~Hz}, 1 \mathrm{H}\right), 1.84\left(\mathrm{dd}, J_{l}=4.0\right.$ $\left.\mathrm{Hz}, J_{2}=12.7 \mathrm{~Hz}, 1 \mathrm{H}\right), 2.80(\mathrm{~m}, 4 \mathrm{H}), 6.56(\mathrm{~d}, J=9.0 \mathrm{~Hz}, 1 \mathrm{H}), 6.96(\mathrm{~d}, J=16.2 \mathrm{~Hz}$, 1H), 7.25-7.34 (m, 3H), $7.53(\mathrm{~s}, 1 \mathrm{H}), 8.17(\mathrm{~s}, 1 \mathrm{H}), 8.30(\mathrm{~s}, 1 \mathrm{H})$. HRMS-EI $(\mathrm{m} / \mathrm{z})$ : $\left[\mathrm{M}-\mathrm{CO}_{2}\right]^{+}$calcd for $\mathrm{C}_{26} \mathrm{H}_{24} \mathrm{~N}_{2} \mathrm{~S}_{3}, 460.1102$; found, 460.1107 .

2-Cyano-3-[5-(2-\{5-[2-(1,2,2,4-tetramethyl-1,2,3,4-tetrahydro-quinolin-6-yl)-vinyl] -thiophen-2-yl \}-vinyl)-thiophen-2-yl]-acrylic acid (C1-5). Black solid (yield 64\%). Mp. $>260{ }^{\circ} \mathrm{C} .{ }^{1} \mathrm{H}-\mathrm{NMR}\left(\right.$ DMSO-d $\left._{6}, 400 \mathrm{MHz}, \mathrm{ppm}\right): \delta 1.17$ (s, 3H), 1.26 (s, 3H), 1.33 $(\mathrm{d}, J=6.6 \mathrm{~Hz}, 3 \mathrm{H}), 1.41\left(\mathrm{dd}, J_{l}=12.8 \mathrm{~Hz}, J_{2}=13.4 \mathrm{~Hz}, 1 \mathrm{H}\right), 1.83\left(\mathrm{dd}, J_{1}=4.1 \mathrm{~Hz}, J_{2}\right.$ $=12.8 \mathrm{~Hz}, 1 \mathrm{H}), 2.79(\mathrm{~m}, 4 \mathrm{H}), 6.53(\mathrm{~d}, J=9.0 \mathrm{~Hz}, 1 \mathrm{H}), 6.84(\mathrm{~d}, J=16.3 \mathrm{~Hz}, 1 \mathrm{H}), 7.04$ (d, $J=3.5 \mathrm{~Hz}, 1 \mathrm{H}), 7.09-7.17(\mathrm{~m}, 2 \mathrm{H}), 7.23-7.35(\mathrm{~m}, 5 \mathrm{H}), 7.75(\mathrm{~s}, 1 \mathrm{H}), 8.20(\mathrm{~s}, 1 \mathrm{H})$. HRMS-EI $(\mathrm{m} / z)$ : $\left[\mathrm{M}-\mathrm{CO}_{2}\right]^{+}$calcd for $\mathrm{C}_{28} \mathrm{H}_{28} \mathrm{~N}_{2} \mathrm{~S}_{2}, 456.1694$; found, 456.1693.

2-Cyano-3-[5-(1,2,2,4-tetramethyl-1,2,3,4-tetrahydro-quinolin-6-yl)-thiophen-2-yl] -acrylic acid (C2-1). Red brown solid (yield 83\%). Mp. 185-190 ${ }^{\circ} \mathrm{C}$. ${ }^{1} \mathrm{H}-\mathrm{NMR}$ (DMSO-d 6 , 400MHz, ppm): $\delta 1.19$ (s, 3H), 1.29 (s, 3H), 1.34 (d, J=6.5 Hz, 3H), 1.43 $\left(\mathrm{dd}, J_{1}=12.4 \mathrm{~Hz}, J_{2}=13.3 \mathrm{~Hz}, 1 \mathrm{H}\right), 1.86\left(\mathrm{dd}, J_{1}=4.2 \mathrm{~Hz}, J_{2}=13.0 \mathrm{~Hz}, 1 \mathrm{H}\right)$, 2.82-2.84 (m, 4H), $6.61(\mathrm{~d}, J=8.4 \mathrm{~Hz}, 1 \mathrm{H}), 7.42-7.45(\mathrm{~m}, 2 \mathrm{H}), 7.49(\mathrm{~d}, J=3.7 \mathrm{~Hz}$, $1 \mathrm{H}), 7.82(\mathrm{~d}, J=3.6 \mathrm{~Hz}, 1 \mathrm{H}), 8.27(\mathrm{~s}, 1 \mathrm{H})$. HRMS-EI $(\mathrm{m} / \mathrm{z}):\left[\mathrm{M}_{-} \mathrm{CO}_{2}\right]^{+}$calcd for $\mathrm{C}_{20} \mathrm{H}_{22} \mathrm{~N}_{2} \mathrm{~S}, 322.1504$; found, 322.1498.

2-Cyano-3-[5'-(1,2,2,4-tetramethyl-1,2,3,4-tetrahydro-quinolin-6-yl)-[2,2']bithiophe nyl-5-yl]-acrylic acid (C2-2). Black solid (yield 67\%). Mp. 195-198 ${ }^{\circ} \mathrm{C} .{ }^{1} \mathrm{H}-\mathrm{NMR}$ (DMSO-d 6 , 400MHz, ppm): $\delta 1.18$ (s, 3H), 1.27 (s, 3H), 1.35 (d, $J=6.7 \mathrm{~Hz}, 3 \mathrm{H})$, $1.43\left(\mathrm{dd}, J_{l}=12.5 \mathrm{~Hz}, J_{2}=13.0 \mathrm{~Hz}, 1 \mathrm{H}\right), 1.85\left(\mathrm{dd}, J_{l}=4.6 \mathrm{~Hz}, J_{2}=13.0 \mathrm{~Hz}, 1 \mathrm{H}\right)$, 
2.80-2.82 (m, 4H), $6.58(\mathrm{~d}, J=8.6 \mathrm{~Hz}, 1 \mathrm{H}), 7.36-7.40(\mathrm{~m}, 3 \mathrm{H}), 7.52(\mathrm{~d}, J=4.1 \mathrm{~Hz}$, 1H), 7.55 (d, $J=3.9 \mathrm{~Hz}, 1 \mathrm{H}), 7.93$ (d, $J=3.6 \mathrm{~Hz}, 1 \mathrm{H}), 8.42$ (s, 1H). HRMS-EI $(\mathrm{m} / \mathrm{z})$ : $\left[\mathrm{M}-\mathrm{CO}_{2}\right]^{+}$calcd for $\mathrm{C}_{24} \mathrm{H}_{24} \mathrm{~N}_{2} \mathrm{~S}_{2}, 404.1381$; found, 404.1387.

2-Cyano-3-[5"-(1,2,2,4-tetramethyl-1,2,3,4-tetrahydro-quinolin-6-yl)-[2,2';5',2"]tert hiophen-5-yl]-acrylic acid (C2-3). Black solid (yield 63\%). Mp.: 196-200 ${ }^{\circ} \mathrm{C}$. ${ }^{1} \mathrm{H}-\mathrm{NMR}$ (DMSO-d 6 , 400MHz, ppm): $\delta 1.18$ (s, 3H), 1.27 (s, 3H), 1.34 (d, $J=6.3 \mathrm{~Hz}$, $3 \mathrm{H}), 1.43\left(\mathrm{dd}, J_{l}=13.2 \mathrm{~Hz}, J_{2}=13.4 \mathrm{~Hz}, 1 \mathrm{H}\right), 1.84\left(\mathrm{dd}, J_{1}=4.3 \mathrm{~Hz}, J_{2}=13.2 \mathrm{~Hz}\right.$, 1H), 2.80-2.82 (m, 4H), 6.58 (d, $J=8.4 \mathrm{~Hz}, 1 \mathrm{H}), 7.29$ (d, $J=4.1 \mathrm{~Hz}, 1 \mathrm{H}), 7.33-7.38$ (m, 4H), $7.55(\mathrm{~m}, 2 \mathrm{H}), 7.88(\mathrm{~d}, J=4.0 \mathrm{~Hz}, 1 \mathrm{H}), 8.36(\mathrm{~s}, 1 \mathrm{H})$. HRMS-EI $(\mathrm{m} / \mathrm{z})$ : $\left[\mathrm{M}-\mathrm{CO}_{2}\right]^{+}$calcd for $\mathrm{C}_{28} \mathrm{H}_{26} \mathrm{~N}_{2} \mathrm{~S}_{3}, 486.1258$; found, 486.1250 .

2-Cyano-3-[6-(1,2,2,4-tetramethyl-1,2,3,4-tetrahydro-quinolin-6-yl)-dithieno[3,2-b; 2',3'-d]thiophen-2-yl]-acrylic acid (C2-4). Black solid (yield 54\%). Mp. 210-213 ${ }^{\circ} \mathrm{C}$. ${ }^{1} \mathrm{H}-\mathrm{NMR}\left(\mathrm{DMSO}_{\mathrm{d}}, 400 \mathrm{MHz}, \mathrm{ppm}\right): \delta 1.19$ (s, 3H), 1.28 (s, 3H), 1.36 (d, $J=6.4 \mathrm{~Hz}$, $3 \mathrm{H}), 1.44\left(\mathrm{dd}, J_{l}=12.1 \mathrm{~Hz}, J_{2}=13.8 \mathrm{~Hz}, 1 \mathrm{H}\right), 1.87\left(\mathrm{dd}, J_{1}=4.6 \mathrm{~Hz}, J_{2}=13.8 \mathrm{~Hz}\right.$, $1 \mathrm{H}), 2.82-2.84(\mathrm{~m}, 4 \mathrm{H}), 6.62(\mathrm{~d}, J=8.9 \mathrm{~Hz}, 1 \mathrm{H}), 7.41(\mathrm{~m}, 2 \mathrm{H}), 7.82(\mathrm{~s}, 1 \mathrm{H}), 8.36(\mathrm{~s}$, $1 \mathrm{H}), 8.55(\mathrm{~s}, 1 \mathrm{H})$. HRMS-EI $(\mathrm{m} / \mathrm{z})$ : $\left[\mathrm{M}-\mathrm{CO}_{2}\right]^{+}$calcd for $\mathrm{C}_{24} \mathrm{H}_{22} \mathrm{~N}_{2} \mathrm{~S}_{3}, 434.0945$; found, 434.0947.

3-\{5-[2-(1-Benzyl-2,2,4-trimethyl-1,2,3,4-tetrahydro-quinolin-6-yl)-vinyl]-thiophe n-2-yl -2-cyano-acrylic acid (C3-1). Dark brown solid (yield 82\%). Mp. 202-207 ${ }^{\circ} \mathrm{C}$. ${ }^{1} \mathrm{H}-\mathrm{NMR}$ (DMSO-d 6 , 400MHz, ppm): $\delta 1.33$ (s, 3H), 1.34 (s, 3H), 1.73 (dd, $J_{l}=13.0$ $\left.\mathrm{Hz}, J_{2}=13.1 \mathrm{~Hz}, 1 \mathrm{H}\right), 1.97\left(\mathrm{dd}, J_{1}=4.5 \mathrm{~Hz}, J_{2}=13.3 \mathrm{~Hz}, 1 \mathrm{H}\right), 3.08(\mathrm{~m}, 4 \mathrm{H}), 4.38(\mathrm{~d}$, $J=18.1 \mathrm{~Hz}, 1 \mathrm{H}), 4.86(\mathrm{~d}, J=18.1 \mathrm{~Hz}, 1 \mathrm{H}), 6.31(\mathrm{~d}, J=8.7 \mathrm{~Hz}, 1 \mathrm{H}), 7.20-7.25(\mathrm{~m}$, 5H), 7.29-7.33 (m, 4H), 7.51 (s, 1H), 7.82 (d, $J=4.0 \mathrm{~Hz}, 1 \mathrm{H}), 8.35$ (s, 1H). HRMS-EI (m/z): [M-CO $]^{+}$calcd for $\mathrm{C}_{28} \mathrm{H}_{28} \mathrm{~N}_{2} \mathrm{~S}, 424.1973$; found, 424.1970. 


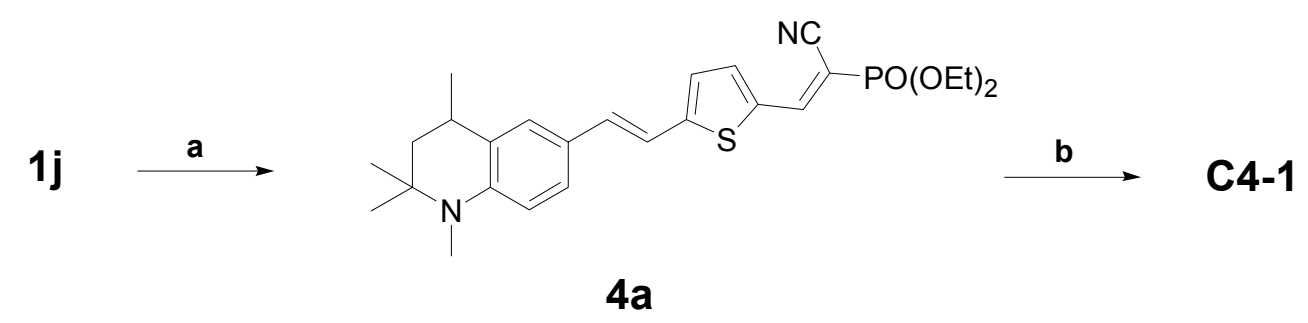

Scheme S4 Synthesis route of C4-1 dye. a) diethyl cyano-methylphosphonate, acetonitrile, piperidine, reflux for $2 \mathrm{~h}, 78 \%$; b) i) trimethylsilane iodide (TMSI), $\mathrm{CH} 2 \mathrm{Cl} 2$, reflux for $1.5 \mathrm{~h}$; ii) $\mathrm{CH}_{3} \mathrm{OH}$, r.t. $1 \mathrm{~h}, 93 \%$.

(1-Cyano-2-\{5-[2-(1,2,2,4-tetramethyl-1,2,3,4-tetrahydro-quinolin-6-yl)-vinyl]-thioph en-2-yl \}-vinyl)-phosphonic acid dithyl ester (4a)

To a solution of $\mathbf{1 j}$ (65 mg, $0.2 \mathrm{mmol}$ ) and diethyl cyanomethyl-phosphonate (43 $\mathrm{mg}, 0.24 \mathrm{mmol}$ ) in $5 \mathrm{~mL}$ acetonitrile, 5 drop of piperidine was added. The reaction mixture was heated to reflux for $2 \mathrm{~h}$ under $\mathrm{N}_{2}$. After removing of solvent the residue was column chromatographied (silica gel, $\mathrm{CH}_{2} \mathrm{Cl}_{2}$ : EtOAc $=10: 1$ as eluent) to give a dark solid (75 mg, 78\%). Mp. 112-113 ${ }^{\circ} \mathrm{C} .{ }^{1} \mathrm{H}-\mathrm{NMR}$ (Acetone-d $\mathrm{d}_{6}, 400 \mathrm{MHz}, \mathrm{ppm}$ ): $\delta$ $1.24(\mathrm{~s}, 3 \mathrm{H}), 1.33(\mathrm{~s}, 3 \mathrm{H}), 1.34-1.39(\mathrm{~m}, 9 \mathrm{H}), 1.47\left(\mathrm{dd}, J_{1}=12.9 \mathrm{~Hz}, J_{2}=13.0 \mathrm{~Hz}\right.$, $1 \mathrm{H}), 1.87\left(\mathrm{dd}, J_{1}=4.4 \mathrm{~Hz}, J_{2}=13.1 \mathrm{~Hz}, 1 \mathrm{H}\right), 2.80-2.89(\mathrm{~m}, 4 \mathrm{H}), 4.14-4.21(\mathrm{~m}, 4 \mathrm{H})$, $6.60(\mathrm{~d}, J=8.5 \mathrm{~Hz}, 1 \mathrm{H}), 7.24-7.25(\mathrm{~m}, 3 \mathrm{H}), 7.37(\mathrm{~d}, J=8.5 \mathrm{~Hz}, 1 \mathrm{H}), 7.44(\mathrm{~s}, 1 \mathrm{H})$, $7.79(\mathrm{~d}, J=4.0 \mathrm{~Hz}, 1 \mathrm{H}), 8.03(\mathrm{~d}, J=19.2 \mathrm{~Hz}, 1 \mathrm{H})$. HRMS-EI $(m / z):[\mathrm{M}]^{+}$calcd for $\mathrm{C}_{26} \mathrm{H}_{33} \mathrm{~N}_{2} \mathrm{O}_{3} \mathrm{PS}$, 484.1949; found, 484.1953.

(1-Cyano-2-\{5-[2-(1,2,2,4-tetramethyl-1,2,3,4-tetrahydro-quinolin-6-yl)-vinyl]-thioph en-2-yl -vinyl)-phosphonic acid (C4-1).

Trimethylsilane iodide $(0.1 \mathrm{~mL})$ was added dropwise to a solution of $\mathbf{4 a}$ (48 $\mathrm{mg}, 0.1$ mmol) in $\mathrm{CH}_{2} \mathrm{Cl}_{2}(10 \mathrm{~mL})$. After refluxing for $1.5 \mathrm{~h}$, the mixture was cooled to $\mathrm{r}$. $\mathrm{t}$. and $\mathrm{CH}_{3} \mathrm{OH}(1 \mathrm{~mL})$ was added. The solvent was removed after stirring for $1 \mathrm{~h}$ and the residue was loaded to a silica gel column chromatography. $\mathrm{CH}_{3} \mathrm{OH}$ was used as eluent and gave brown solid (yield 93\%). Mp.: 201-203 ${ }^{\circ} \mathrm{C} .{ }^{1} \mathrm{H}-\mathrm{NMR}$ (DMSO-d $\mathrm{d}_{6}, 400 \mathrm{MHz}$, ppm): $\delta 1.16(\mathrm{~s}, 3 \mathrm{H}), 1.26(\mathrm{~s}, 3 \mathrm{H}), 1.32(\mathrm{~d}, J=5.8 \mathrm{~Hz}, 3 \mathrm{H}), 1.40\left(\mathrm{dd}, J_{l}=12.4 \mathrm{~Hz}, J_{2}\right.$ $=13.4 \mathrm{~Hz}, 1 \mathrm{H}), 1.82\left(\mathrm{dd}, J_{1}=4.0 \mathrm{~Hz}, J_{2}=12.8 \mathrm{~Hz}, 1 \mathrm{H}\right), 2.78(\mathrm{~m}, 4 \mathrm{H}), 6.51(\mathrm{~d}, J=$ 8.6 Hz, 1H), $6.93(\mathrm{~d}, J=16.0 \mathrm{~Hz}, 1 \mathrm{H}), 7.09(\mathrm{~s}, 1 \mathrm{H}), 7.16(\mathrm{~d}, J=16.1 \mathrm{~Hz}, 1 \mathrm{H}), 7.25$ (d, $J=8.5 \mathrm{~Hz}, 1 \mathrm{H}), 7.31(\mathrm{~s}, 1 \mathrm{H}), 7.41(\mathrm{~s}, 1 \mathrm{H}), 7.64(\mathrm{~d}, J=14.5 \mathrm{~Hz}, 1 \mathrm{H})$. HRMS-EI $(\mathrm{m} / \mathrm{z})$ : $\left[\mathrm{M}-\mathrm{PO}_{3} \mathrm{H}\right]^{+}$calcd for $\mathrm{C}_{22} \mathrm{H}_{24} \mathrm{~N}_{2} \mathrm{~S}, 348.1660$; found, 348.1665 . 
Reference:

1. S. Inaoka, D. M. Collard, J. Mater. Chem, 1999, 9, 1719-1725.

2. M. J. Janssen, F. De Jong, J. Org. Chem. 1971, 36, 1645-1648.

3. R. E. Müller, F. F. Nord, J. Org. Chem. 1951, 16, 1380-1388.

4. J. D. Surmatis, A. Ofner, J. Org. Chem. 1963, 28, 2735-2739.

5. T. Mitsumori, K. Inoue, N. Koga, H. Iwamura, J. Am. Chem. Soc. 1995, 117, 2467-2478.

6. F. Ellinger, A. Gieren, Th. Huebner, J. Lex, F. Lucchesini, et al., Monatsh. Chem. $1993,124,931-944$.

7. O. -K. Kim, A. Fort, M. Barzoukas, M. Blanchard-Desce, J. -M. Lehn, J. Mater. Chem. 1999, 9, 2227-2232.

8. G. Kossmehl, D. Budwill, Z. Naturforsch. B 1987, 42, 478-488.

9. J. -M. Raimundo, P. Blanchard, N. Gallego-Planas, N. Mercier, I. Ledoux-Rak, R. Hierle, J. Roncali, J. Org. Chem. 2002, 67, 205-218.

10. J. Nakayama, Y. Nakamura, T. Tajiri, Heterocycles, 1986, 24, 637-640

11. Stoyanovich, Fedorov, Zh. Org. Khim. 1965, 1, 1282.

12. D. Maurizio, M. Antonella De, D. Franco, P. Giovanni, Synth. Commun. 1987, 17, 491-497.

13. J. P. Parakka, M. P. Cava, Tetrahedron; 1995, 51, 2229-2242. 
(B) Photophysical properties of the tetrahydroquinoline sensitizers:
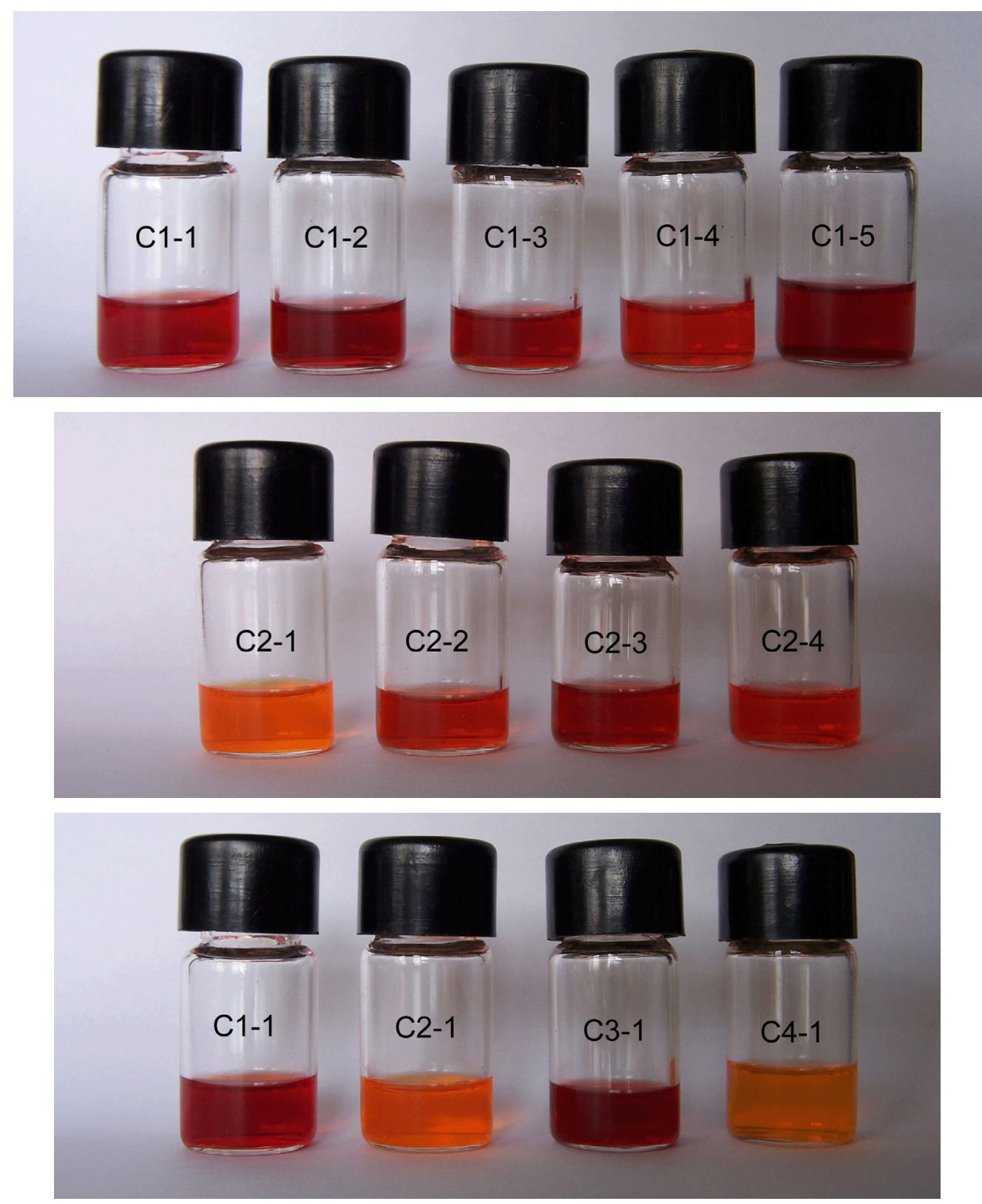

Figure S1. Image of the dye solutions. 

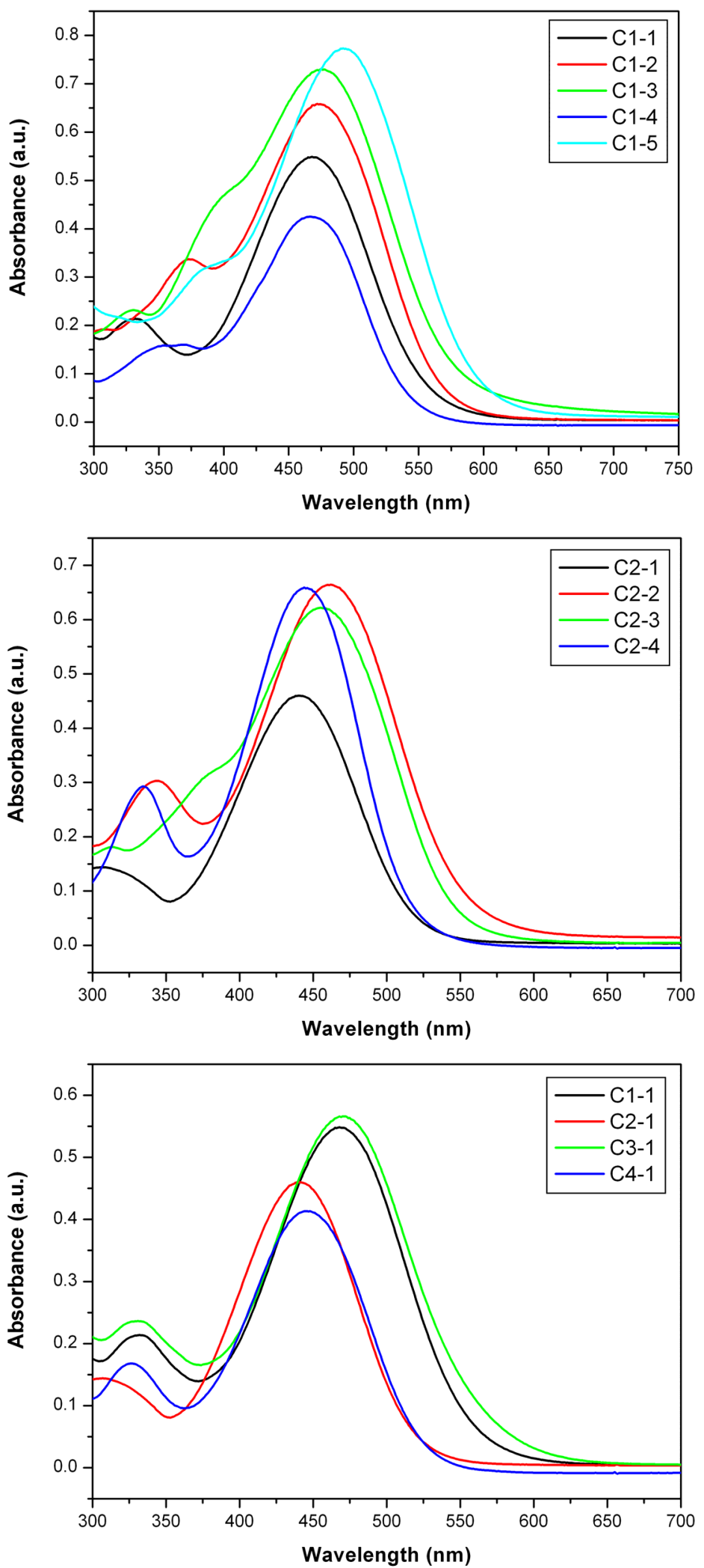

Figure S2 UV-vis spectra of the dyes in ethanol solutions $\left(2 \times 10^{-5} \mathrm{M}\right)$ at $25^{\circ} \mathrm{C} . \mathbf{C 1 - 4}, \mathbf{C 2 - 3}$ and C2-4 were measured in DMF solutions $\left(2 \times 10^{-5} \mathrm{M}\right)$ at $25^{\circ} \mathrm{C}$. 

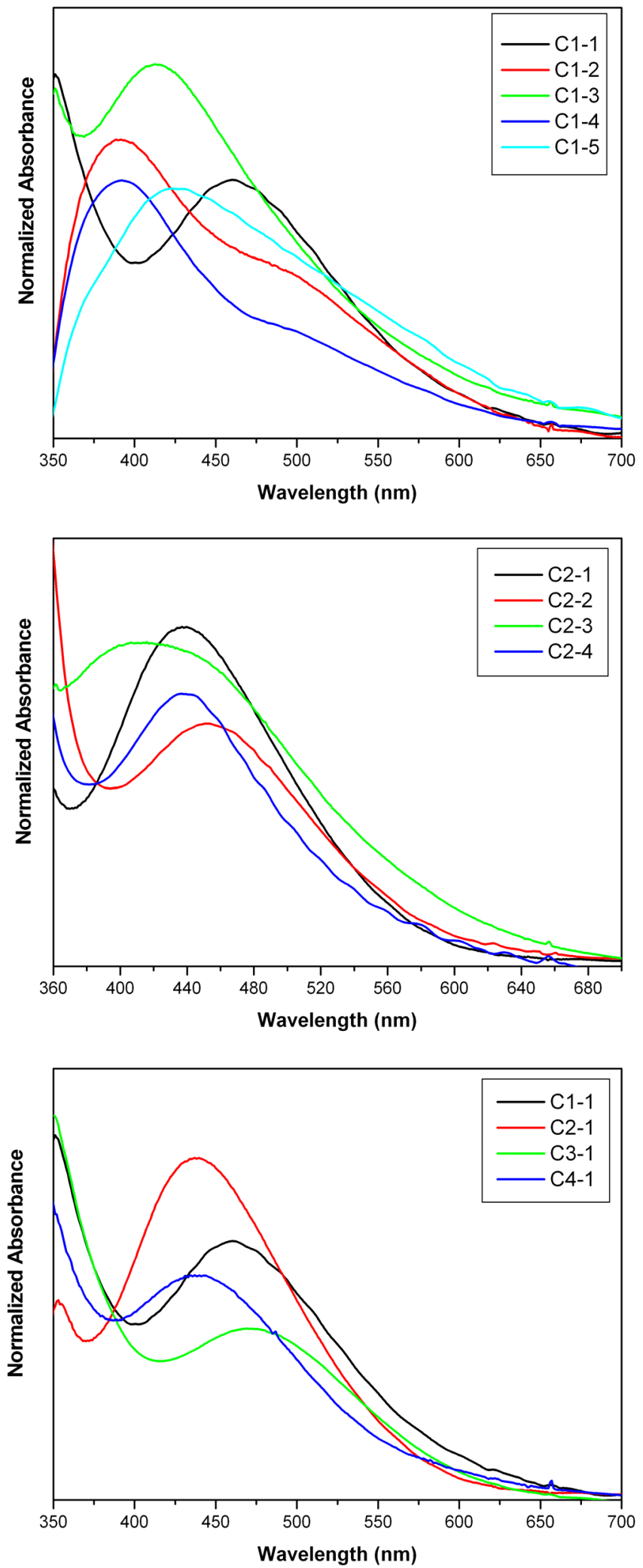

Figure S3. Absorption spectra of the dyes anchored on $\mathrm{TiO}_{2}$ surface. 

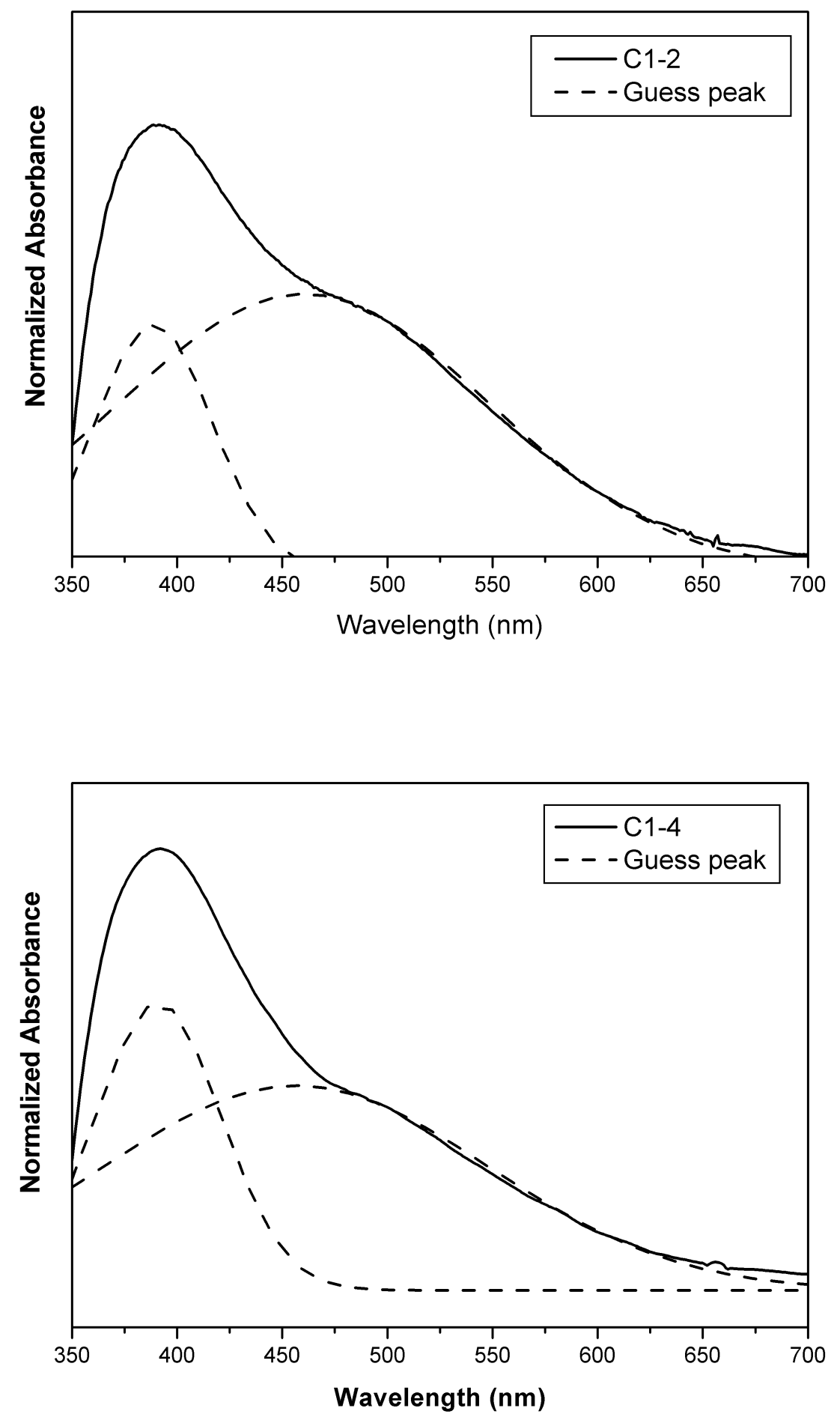

Figure S4 Absorption spectra and curve fitting of C1-2 and C1-4 dyes anchored on $\mathrm{TiO}_{2}$. 

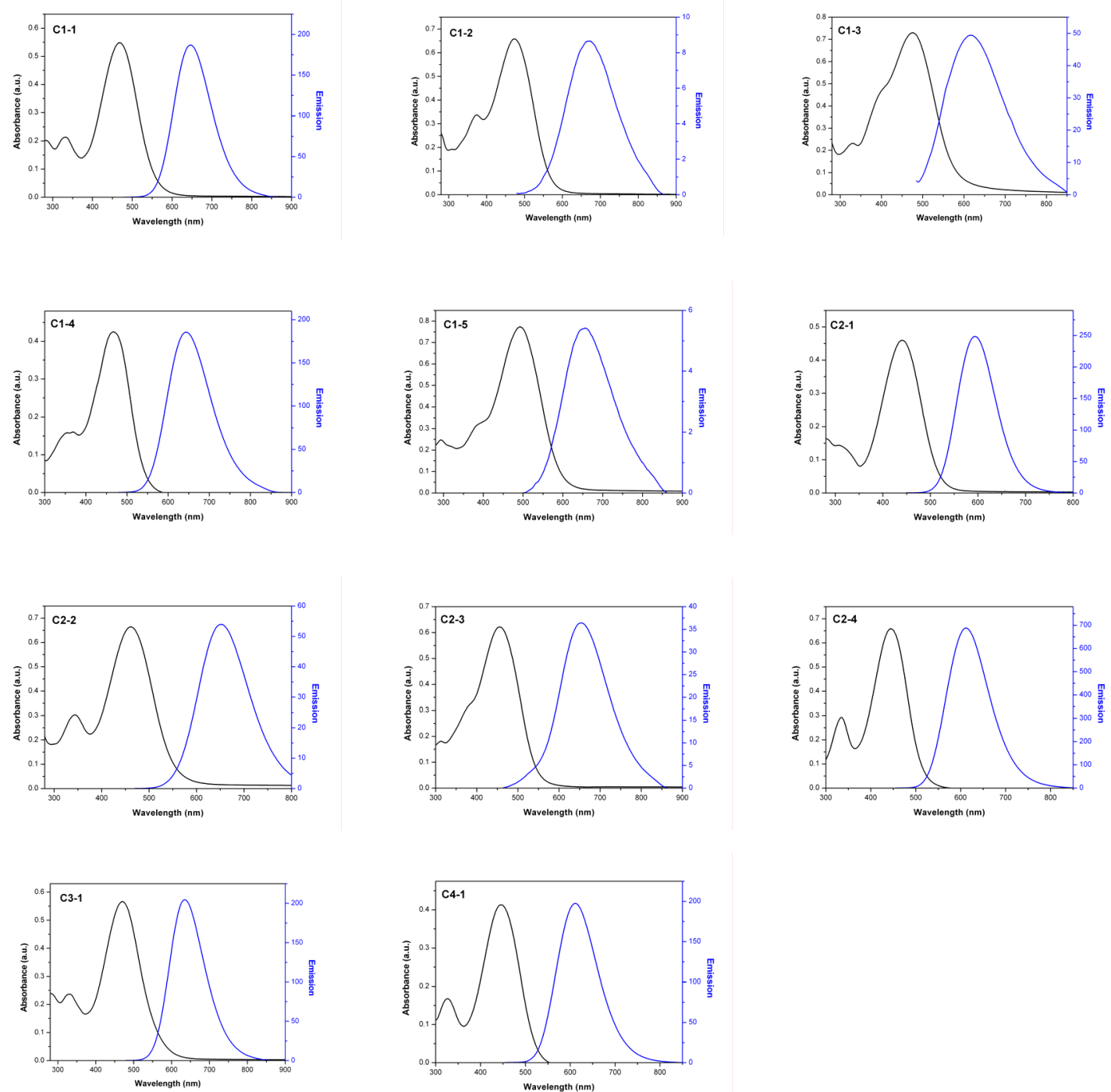

Figure S5. Absorption (black) and emission (blue) spectra of the dyes in ethanol solutions; C1-4, C2-3 and C2-4 were measured in DMF solutions. 


\section{(C) Characterization of the DSSCs:}

Table S1 Photovoltaic performance of DSSCs based on the tetrahydroquinoline dyes with and without the addition of $\mathrm{CDCA}^{[\mathrm{a}]}$

\begin{tabular}{|c|c|c|c|c|}
\hline Dye & $\begin{array}{c}J_{s c} \\
\left(\mathrm{~mA} / \mathrm{cm}^{2}\right)\end{array}$ & $V_{o c}(\mathrm{mV})$ & Fill factor $(f f)$ & $\eta(\%)$ \\
\hline C1-1 ${ }^{[\mathrm{b}]}$ & 7.80 & 582 & 0.65 & 2.93 \\
\hline C1-1 $\mathrm{CDCA}^{[\mathrm{b}]}$ & 8.48 & 583 & 0.64 & 3.17 \\
\hline C1-2 & 5.01 & 516 & 0.60 & 1.56 \\
\hline C1-2 CDCA & 6.99 & 543 & 0.62 & 2.35 \\
\hline C1-3 & 4.68 & 500 & 0.56 & 1.32 \\
\hline C1-3 CDCA & 6.39 & 521 & 0.57 & 1.91 \\
\hline $\mathrm{C} 1-4^{[\mathrm{c}]}$ & 6.38 & 495 & 0.56 & 1.78 \\
\hline $\mathbf{C 1 - 4} \mathrm{CDCA}^{[\mathrm{c}]}$ & 6.87 & 514 & 0.66 & 2.34 \\
\hline C1-5 & 3.39 & 532 & 0.66 & 1.19 \\
\hline C1-5 CDCA & 7.22 & 542 & 0.59 & 2.32 \\
\hline C2-1 ${ }^{[\mathrm{b}]}$ & 11.3 & 600 & 0.66 & 4.47 \\
\hline $\mathbf{C} \mathbf{2}-\mathbf{1} \mathrm{CDCA}^{[\mathrm{b}]}$ & 11.2 & 600 & 0.67 & 4.49 \\
\hline $\mathrm{C} 2-2$ & 9.82 & 590 & 0.65 & 3.74 \\
\hline C2-2 CDCA & 12.0 & 597 & 0.63 & 4.53 \\
\hline $\mathbf{C 2 - 3 ^ { [ c ] }}$ & 10.00 & 537 & 0.64 & 3.44 \\
\hline $\mathbf{C 2 - 3} \mathrm{CDCA}^{[\mathrm{c}]}$ & 8.53 & 527 & 0.67 & 3.00 \\
\hline $\mathrm{C} 2-4^{[\mathrm{c}]}$ & 8.35 & 524 & 0.63 & 2.77 \\
\hline $\mathbf{C 2 - 4} \mathrm{CDCA}^{[\mathrm{c}]}$ & 8.84 & 522 & 0.63 & 2.92 \\
\hline C3-1 ${ }^{[\mathrm{b}]}$ & 7.50 & 560 & 0.64 & 2.70 \\
\hline C3-1 $\mathrm{CDCA}^{[\mathrm{b}]}$ & 9.27 & 580 & 0.67 & 3.61 \\
\hline C4-1 & 4.79 & 554 & 0.67 & 1.79 \\
\hline C4-1 CDCA & 8.54 & 568 & 0.67 & 3.27 \\
\hline $\mathrm{N} 3^{[\mathrm{d}]}$ & 14.03 & 695 & 0.63 & 6.16 \\
\hline
\end{tabular}

[a] Conditions: Irradiated light: AM1.5 $\left(100 \mathrm{~mW} / \mathrm{cm}^{2}\right)$; $\mathrm{TiO}_{2}$ films thickness: $10 \mu \mathrm{m}$; Dye bath: ethanol solutions $\left(1 \times 10^{-4} \mathrm{M}\right)$ with or without the addition of chenodexoycholic acid $\left(\mathrm{CDCA}, 3 \times 10^{-3} \mathrm{M}\right)$; Working area: $0.159 \mathrm{~cm}^{2}$; Electrolyte: 0.6 M 1,2-dimethyl-3-n-propylimidazolium iodide/ $0.1 \mathrm{M}$ LiI/ $0.05 \quad \mathrm{M}_{2} / 0.5 \mathrm{M}$ 4-tert-butylpyridine in 3-methoxyproponitrile. [b] Dye bath: ethanol solution $\left(2 \times 10^{-4}\right.$ $\mathrm{M})$ with or without the addition of CDCA $\left(3 \times 10^{-3} \mathrm{M}\right)$; [c] Dye bath: DMF solution $\left(1 \times 10^{-4} \mathrm{M}\right)$ with or without the addition of CDCA $\left(3 \times 10^{-3} \mathrm{M}\right)$; [d] Dye bath: ethanol solution $\left(3 \times 10^{-4} \mathrm{M}\right)$. 

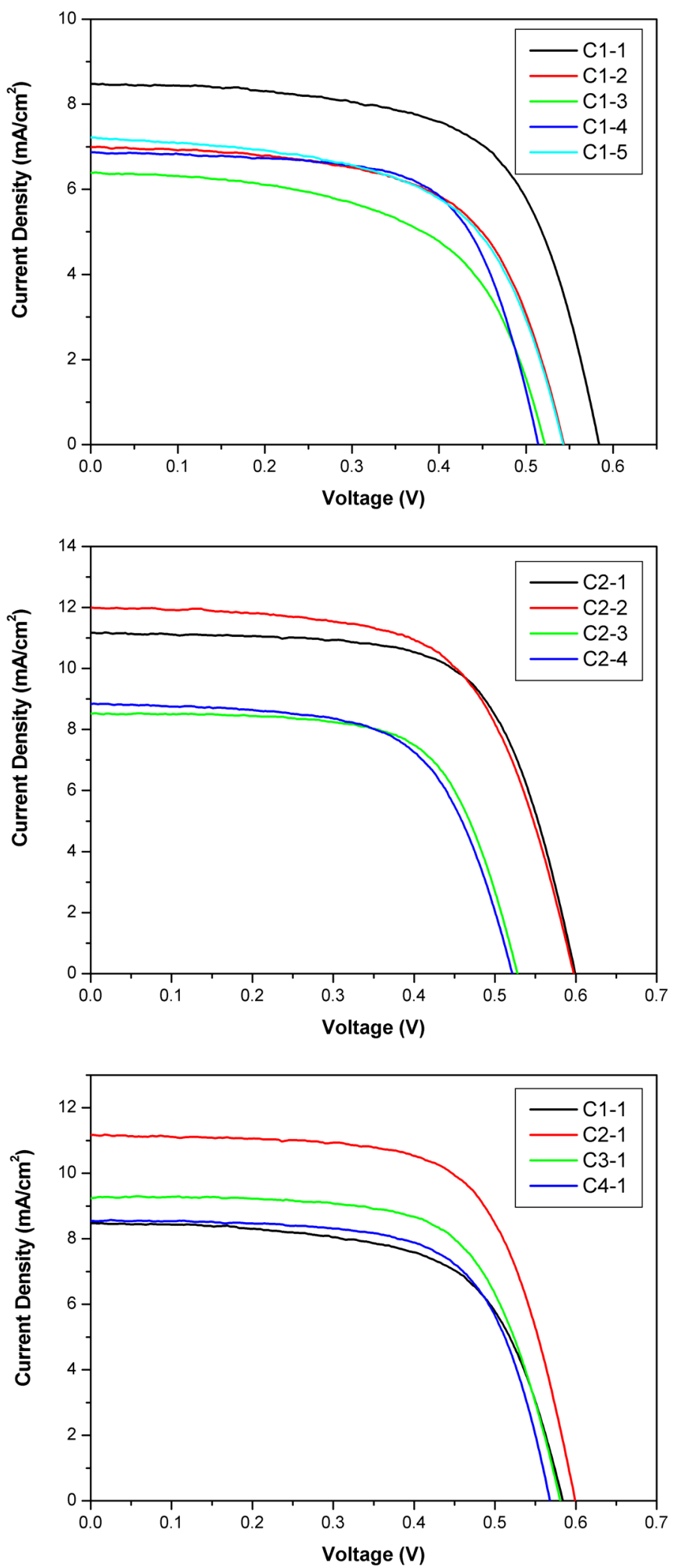

Figure S6 $J-V$ curves of DSSCs based on tetrahydroquinoline dyes with the addition of CDCA into dye bath. 

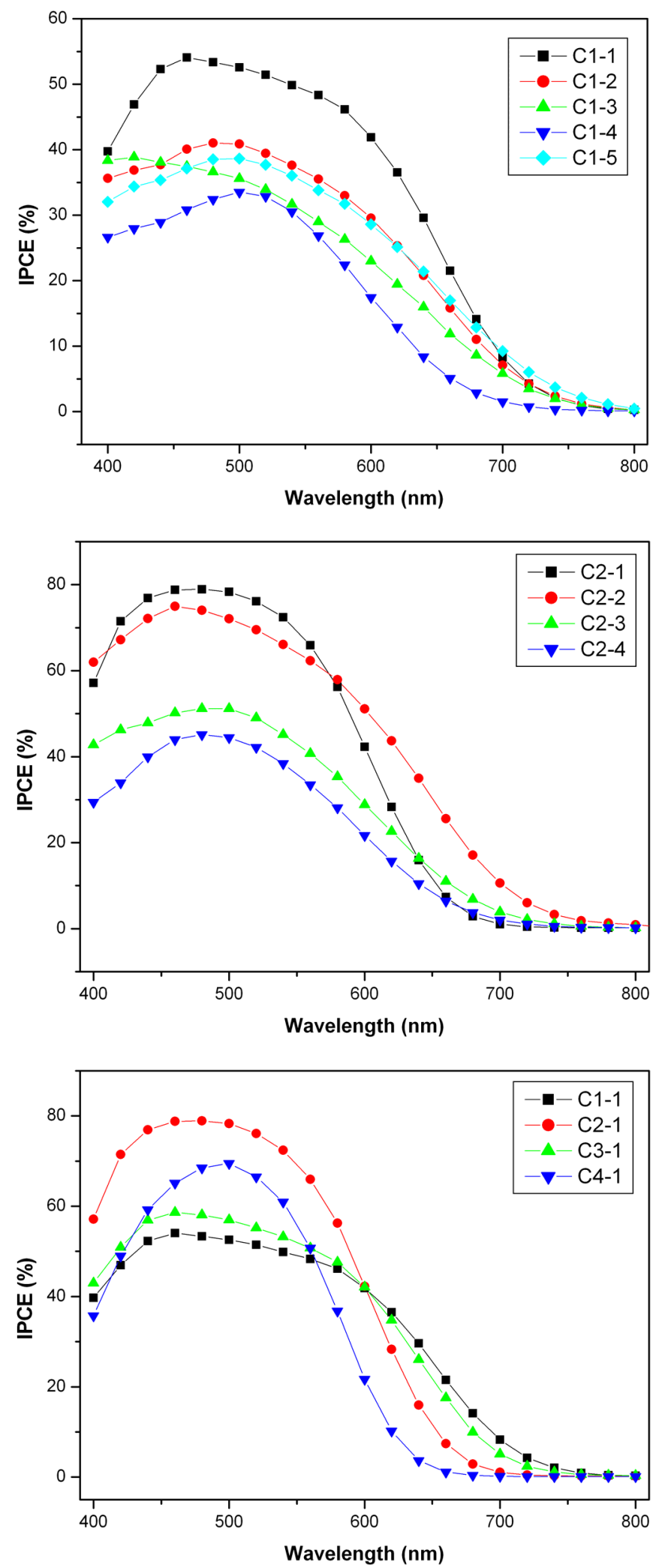

Figure S7 Incident photon-to-current conversion efficiencies of the DSSCs based on tetrahydroquinoline dyes. 

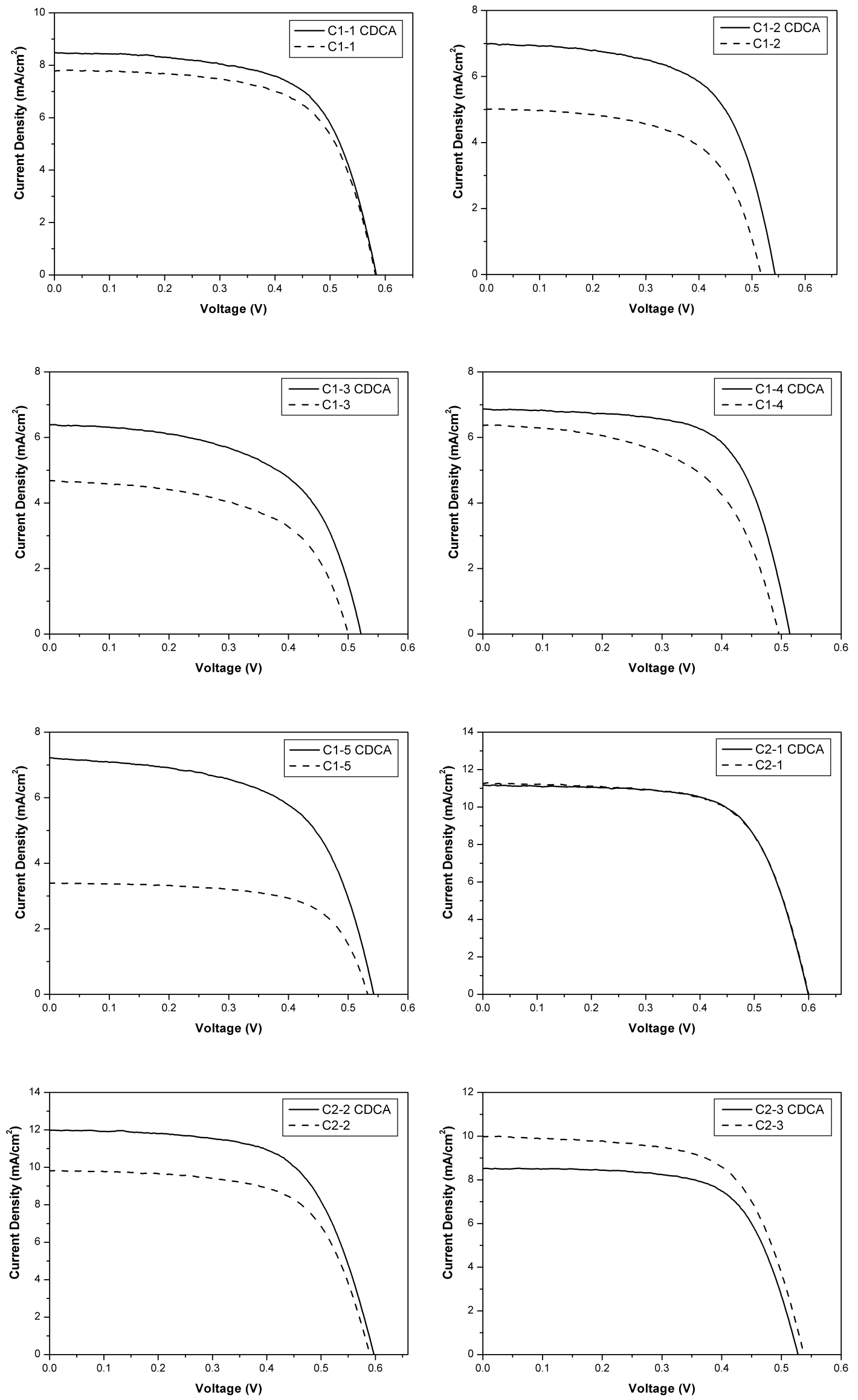

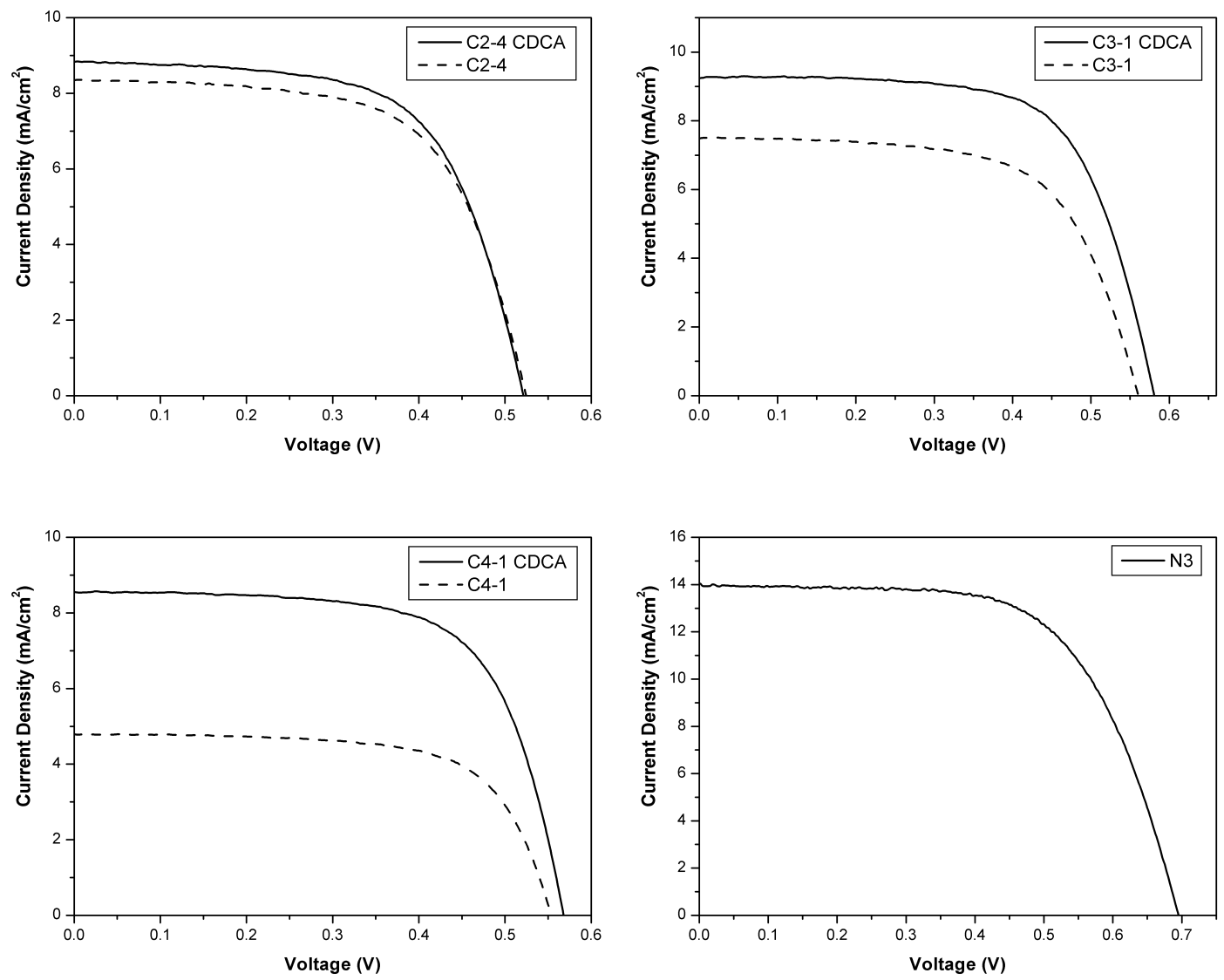

Figure S8 $J-V$ curves of DSSCs based on the tetrahydroquinoline dyes with (-) or without (- - ) the addition of $\operatorname{CDCA}\left(3 \times 10^{-3} \mathrm{M}\right)$ into dye bath. 


\section{(D) Complete Reference 38}

38. GAUSSIAN 03, Revision B.03, Frisch, M. J.; Trucks, G. W.; Schlegel, H. B.; Scuseria, G. E.; Robb, M. A.; Cheeseman, J. R.; Montgomery, Jr., J. A.; Vreven, T.;

Kudin, K. N.; Burant, J. C.; Millam, J. M.; Iyengar, S. S.; Tomasi, J.; Barone, V.; Mennucci, B.; Cossi, M.; Scalmani, G.; Rega, N.; Petersson, G. A.; Nakatsuji, H.; Hada, M.; Ehara, M.; Toyota, K.; Fukuda, R.; Hasegawa, J.; Ishida, M.; Nakajima, T.; Honda, Y.; Kitao, O.; Nakai, H.; Klene, M.; Li, X.; Knox, J. E.; Hratchian, H. P.; Cross, J. B.; Bakken, V.; Adamo, C.; Jaramillo, J.; Gomperts, R.; Stratmann, R. E.; Yazyev, O.; Austin, A. J.; Cammi, R.; Pomelli, C.; Ochterski, J. W.; Ayala, P. Y.; Morokuma, K.; Voth, G. A.; Salvador, P.; Dannenberg, J. J.; Zakrzewski, V. G.; Dapprich, S.; Daniels, A. D.; Strain, M. C.; Farkas, O.; Malick, D. K.; Rabuck, A. D.; Raghavachari, K.; Foresman, J. B.; Ortiz, J. V.; Cui, Q.; Baboul, A. G.; Clifford, S.; Cioslowski, J.; Stefanov, B. B.; Liu, G.; Liashenko, A.; Piskorz, P.; Komaromi, I.; Martin, R. L.; Fox, D. J.; Keith, T.; Al-Laham, M. A.; Peng, C. Y.; Nanayakkara, A.; Challacombe, M.; Gill, P. M. W.; Johnson, B.; Chen, W.; Wong, M. W.; Gonzalez, C.; and Pople, J. A.; Gaussian, Inc., Pittsburgh PA, 2003. 